\title{
Pericytes regulate vascular immune homeostasis in the CNS
}

\section{Journal Article}

\section{Author(s):}

Török, Orsolya; Schreiner, Bettina; Schaffenrath, Johanna; Tsai, Hsing-Chuan; Maheshwari, Upasana; Stifter, Sebastian A.; Welsh, Christina; Amorim, Ana; Sridhar, Sucheta; Utz, Sebastian G.; Mildenberger, Wiebke; Nassiri, Sina; Delorenzi, Mauro; Aguzzi, Adriano; Han, May H.; Greter, Melanie; Becher, Burkhard; Keller, Annika

\section{Publication date:}

2021-03-09

\section{Permanent link:}

https://doi.org/10.3929/ethz-b-000475206

\section{Rights / license:}

Creative Commons Attribution-NonCommercial-NoDerivatives 4.0 International

\section{Originally published in:}

Proceedings of the National Academy of Sciences of the United States of America 118(10), https://doi.org/10.1073/ pnas. 2016587118 


\title{
Pericytes regulate vascular immune homeostasis in the CNS
}

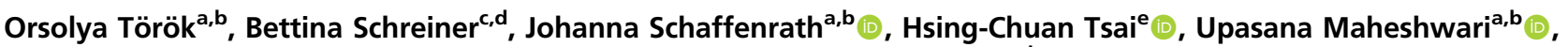

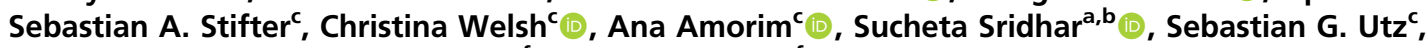 \\ Wiebke Mildenberger ${ }^{c}$, Sina Nassiri ${ }^{f}$, Mauro Delorenzi ${ }^{f}$, Adriano Aguzzi ${ }^{9}{ }^{\circ}$, May H. Han $^{\mathrm{e}}$, Melanie Greter ${ }^{\mathrm{c}}{ }^{\circ}$, \\ Burkhard Becherc ${ }^{\mathbb{D}}$, and Annika Keller ${ }^{\mathrm{a}, \mathrm{b}, 1} \mathbb{\infty}$
}

\begin{abstract}
${ }^{a}$ Department of Neurosurgery, Clinical Neuroscience Center, University Hospital Zürich, Zürich University, 8091 Zürich, Switzerland; ${ }^{b}$ Neuroscience Center Zürich, University of Zürich and ETH Zürich, 8057 Zürich, Switzerland; ' Institute of Experimental Immunology, University of Zürich, 8057 Zürich Switzerland; 'Department of Neurology, University Hospital Zurich, 8091 Zürich, Switzerland; ${ }^{e}$ Department of Neurology and Neurological Sciences, Stanford University, Stanford, CA 94305; ' Bioinformatics Core Facility, Swiss Institute of Bioinformatics, 1015 Lausanne, Switzerland; and Institute of Neuropathology, University Hospital Zürich, 8091 Zürich, Switzerland
\end{abstract}

Edited by Lawrence Steinman, Stanford University School of Medicine, Stanford, CA, and approved January 26, 2021 (received for review August 16, 2020)

Pericytes regulate the development of organ-specific characteristics of the brain vasculature such as the blood-brain barrier (BBB) and astrocytic end-feet. Whether pericytes are involved in the control of leukocyte trafficking in the adult central nervous system (CNS), a process tightly regulated by CNS vasculature, remains elusive. Using adult pericyte-deficient mice $\left(P d g f b^{\text {retret }}\right)$, we show that pericytes limit leukocyte infiltration into the CNS during homeostasis and autoimmune neuroinflammation. The permissiveness of the vasculature toward leukocyte trafficking in Pdgfb ${ }^{\text {retret }}$ mice inversely correlates with vessel pericyte coverage. Upon induction of experimental autoimmune encephalomyelitis (EAE), pericyte-deficient mice die of severe atypical EAE, which can be reversed with fingolimod, indicating that the mortality is due to the massive influx of immune cells into the brain. Additionally, administration of anti-VCAM-1 and anti-ICAM-1 antibodies reduces leukocyte infiltration and diminishes the severity of atypical EAE symptoms of Pdgfb ${ }^{\text {retret }}$ mice, indicating that the proinflammatory endothelium due to absence of pericytes facilitates exaggerated neuroinflammation. Furthermore, we show that the presence of myelin peptide-specific peripheral T cells in Pdgfb ${ }^{\text {retret }} ; 2 D 2^{\text {tg }}$ mice leads to the development of spontaneous neurological symptoms paralleled by the massive influx of leukocytes into the brain. These findings indicate that intrinsic changes within brain vasculature can promote the development of a neuroinflammatory disorder.

pericyte | blood-brain barrier | leukocyte trafficking |

autoimmune neuroinflammation $\mid \mathrm{MOG}_{35-55}$-specific $\mathrm{T}$ cell receptor

$\mathrm{C}_{\mathrm{c}}$ entral nervous system (CNS) vasculature possesses specific features collectively referred to as the blood-brain barrier (BBB), which localizes to endothelial cells. The BBB ensures the delivery of essential nutrients while preventing the entry of xenobiotics into the brain. In addition, brain endothelial cells restrict the invasion of leukocytes into the brain parenchyma, thus contributing to the immune privilege of the CNS. BBB function is induced by neural tissue and established by all cell types constituting the neurovascular unit (NVU). Pericytes and mural cells residing on the abluminal side of capillaries and postcapillary venules regulate several features of the BBB $(1,2)$. Studies on Pdgfb and $P d g f r b$ mouse mutants, which exhibit variable pericyte loss, have demonstrated that pericytes negatively regulate endothelial transcytosis, which, if not suppressed, leads to increased BBB permeability to plasma proteins $(1,2)$. In addition, pericyte-deficient vessels show abnormal astrocyte end-feet polarization (1). Thus, pericytes regulate several characteristics of the brain vasculature during development and in the adult organism $(1,2)$. Whether the nonpermissive properties of the brain vasculature to leukocyte trafficking in the adult organism are regulated by pericytes has not been addressed. Interestingly, increasing evidence points to the role of pericytes in leukocyte extravasation in peripheral organs such as the skin and the striated muscle and in tumors (3-5).
Increased vascular permeability to plasma proteins and immune cells accompanies neurological disorders such as multiple sclerosis (MS), stroke, and Alzheimer's disease (reviewed in refs. $6-8$ ). In MS, a chronic inflammatory and degenerative neurological disorder (9), autoreactive lymphocytes infiltrate the CNS parenchyma, leading to focal inflammatory infiltrates, demyelination, axonal damage, and neurodegeneration. These infiltrating immune cells could induce vascular dysfunction, including permeability to plasma proteins such as fibrinogen $(10-12)$. On the contrary, disruption of the BBB has been shown to precede the infiltration of inflammatory cells and the formation of demyelinating lesions in MS patients (13). Therefore, it is important to understand roles of different cell types and alterations in cell-cell communication at the NVU, which may facilitate entry of autoimmune $\mathrm{T}$ cells as well as the anatomical localization of lesions. However, knowledge about how pericytes contribute to the development of the disease is still limited.

This prompted us to investigate whether pericytes, which regulate several aspects of the BBB phenotype of endothelial cells, also regulate immune cell trafficking into the CNS during homeostasis and neuroinflammation. We show that pericytes play a crucial role

\section{Significance}

The CNS vasculature tightly regulates the passage of circulating molecules and leukocytes into the CNS. In the neuroinflammatory disease multiple sclerosis (MS), these regulatory mechanisms fail, and autoreactive T cells invade the CNS via blood vessels, leading to neurological deficits depending on where the lesions are located. The region-specific mechanisms directing the development of such lesions are not well understood. In this study, we investigated whether pericytes regulate CNS endothelial cell permissiveness toward leukocyte trafficking into the brain parenchyma. By using a pericyte-deficient mouse model, we show that intrinsic changes in the brain vasculature due to absence of pericytes facilitate the neuroinflammatory cascade and can influence the localization of the neuroinflammatory lesions.

Author contributions: O.T., B.S., M.H.H., M.G., B.B., and A.K. designed research; O.T., B.S., J.S., H.-C.T., U.M., S.A.S., C.W., A. Amorim, S.S., S.G.U., W.M., and A.K. performed research; M.G. and B.B. contributed new reagents/analytic tools; O.T., B.S., J.S., U.M., S.A.S., C.W., A. Amorim, S.S., S.G.U., S.N., M.D., A. Aguzzi, M.H.H., M.G., and A.K. analyzed data; and O.T. and A.K. wrote the paper.

The authors declare no competing interest.

This article is a PNAS Direct Submission.

This open access article is distributed under Creative Commons Attribution-NonCommercialNoDerivatives License 4.0 (CC BY-NC-ND).

${ }^{1}$ To whom correspondence may be addressed. Email: annika.keller@usz.ch.

This article contains supporting information online at https://www.pnas.org/lookup/suppl/ doi:10.1073/pnas.2016587118/-/DCSupplemental.

Published March 2, 2021. 
in the regulation of BBB features related to the restricted leukocyte trafficking into the CNS parenchyma, both under physiological and pathophysiological conditions. We show that the permissiveness to leukocyte trafficking into the CNS inversely correlates with the vessel pericyte coverage, suggesting that vascular inflammation of the CNS due to alterations in the cellular composition of the NVU can direct the spatial distribution of neuroinflammation.

\section{Results}

Increased Expression of Leukocyte Adhesion Molecules in the Brain Vasculature in Pericyte-Deficient Adult Mice. To address the question of whether pericytes regulate immune cell trafficking into the $\mathrm{CNS}$, we used a pericyte-deficient mouse line, $P d g f b^{\text {ret/ret }}$, which shows $\sim 90 \%$ reduction in brain pericyte numbers and $\sim 75 \%$ reduction in pericyte vessel coverage in adult animals (1). Earlier studies have shown that pericyte deficiency in embryos leads to increased mRNA levels of leukocyte adhesion molecules (LAMs) on endothelial cells (2). We analyzed published microarray data of the adult $P d g f b^{\text {ret/ret }}$ brain microvasculature (1) and detected a deregulation of several LAMs, including vascular cell adhesion molecule 1 (VCAM-1; $\log 2=0.43, P=0.007)$, in the adult brain microvasculature abated in pericyte numbers (SI Appendix, Fig. $\mathrm{S} 1 A$ ). To corroborate these findings, we investigated whether strongly reduced pericyte coverage in adult mice leads to changes of LAMs at the protein level. We focused on the expression of VCAM-1 and intercellular adhesion molecule 1 (ICAM-1), which play a major role in the cascade of immune cell transmigration into tissues (14). We detected a zonated endothelial expression of both LAMs in control mice (Fig. $1 A$ and $B$ ), similar to a published study (15). In brains of Pdgf $b^{\text {ret } / r e t}$ mice, the zonated expression pattern was lost and paralleled by a conspicuously stronger staining of VCAM-1 and ICAM-1 (Fig. $1 A$ and $B$ ), which colocalized with the endothelial marker podocalyxin (Fig. $1 C$ and $D$ ). Quantification of VCAM-1 and ICAM-1 vessel surface coverage in the cerebral cortex and in the striatum showed a significant increase of VCAM-1 and ICAM-1 expression in Pdgf $b^{\text {rettret }}$ mice compared with controls (Fig. 1E). Thus, pericyte deficiency results in an increased expression of LAMs on the brain vasculature in adult mice.

Leukocyte Extravasation into the Brain in Pericyte-Deficient Adult Mice. We next asked whether increased expression of LAMs on the brain endothelium of pericyte-deficient mice is accompanied
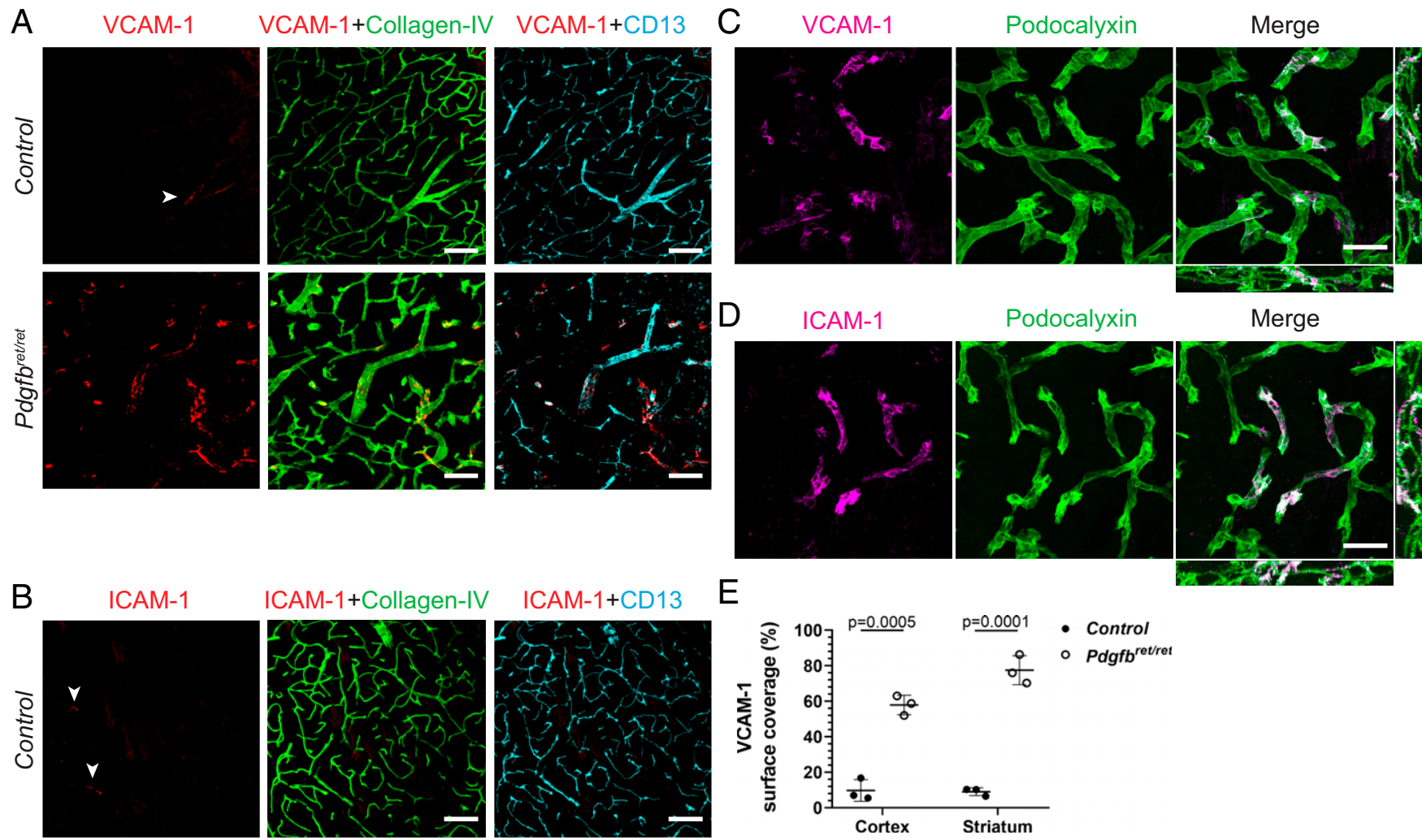

E
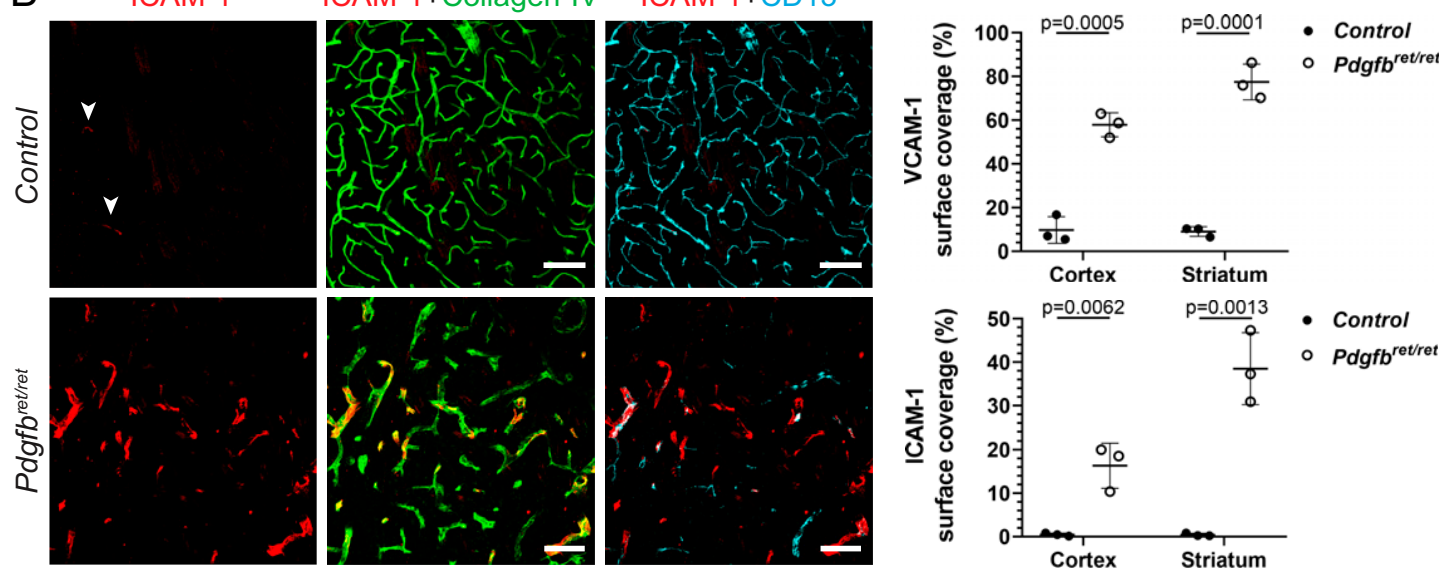

Fig. 1. Increased expression of LAMs on pericyte-deficient brain vasculature. $(A-D)$ Immunofluorescent stainings showing the expression of VCAM-1 (A) and

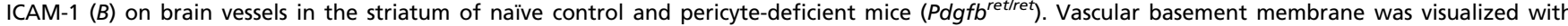
collagen IV (in green) and pericytes with CD13 (in cyan) immunostaining. High-magnification images of the vasculature of Pdgfb ${ }^{\text {ret/ret }}$ mice showing colocalization of VCAM-1 $(C)$ and ICAM-1 $(D)$ staining (in magenta) with the endothelial marker podocalyxin (in green). ( $E$ ) Quantification of vascular surface coverage of VCAM-1 and ICAM-1 in the cortex and striatum in control and pericyte-deficient mice (Pdgfb ${ }^{\text {ret/ret}} ; n=3$ mice per genotype). Unpaired $t$ test was used to determine statistical significance. Data are presented as the mean $\pm \mathrm{SD}$. (Scale bars: $A$ and $B, 100 \mu \mathrm{m} ; C$ and $D, 50 \mu \mathrm{m}$.) 
by leukocyte infiltration into the brain parenchyma. We first analyzed the presence of CD45 $5^{\text {hi }}$ leukocytes in different anatomical regions of the brain by immunofluorescent staining and confocal imaging. Adult $P d g f b^{\text {rettret }}$ mice showed numerous CD $45^{\text {hi }}$ leukocyte infiltrates in the brain parenchyma (Fig. 2A). High-magnification images revealed that $\mathrm{CD} 45^{\text {hi }}$ cells were found in the vessel lumen and in the brain parenchyma and clustered around blood vessels in the brains of $P d g f b^{\text {ret/ret }}$ mice, whereas, in control mice, CD $45^{\text {hi }}$ cells were detected, but they resided in the lumen of blood vessels (Fig. 2 $B$ and $D)$. CD $45^{\text {hi }}$ leukocyte infiltrates can be distinguished from $\mathrm{CD} 45^{\text {lo }}$ microglia based on their round morphology and the lack of expression of the microglia marker P2Y12 (SI Appendix, Fig. S2A). Microglia surrounding $\mathrm{CD} 45^{\text {hi }}$ infiltrates in corpus callosum in $P d g f b^{\text {rettret }}$ mice show a slightly altered morphology of shorter and thicker dendrites (SI Appendix, Fig. S2A), and occasional local alteration in microglia morphology was also detected in the cerebral cortex (SI Appendix, Fig. S2B). Pdgf $b^{\text {rettret }}$ mice show a slightly increased number of microglia in the brain but not in the spinal cord (SI Appendix, Fig. S2 $C$ and D). Although all cerebral regions in $P d g f b^{r e t / r e t}$ mice showed altered vascular expression of LAMs and immune cell infiltrates, quantification of extravasated leukocytes in different brain regions showed that corpus callosum and striatum contained more transmigrated CD $45^{\text {hi }}$ cells compared with cortex (Fig. 2C). Notably, immunohistochemical analysis of CD45 hi cells in the spinal cord of $P d g f b^{\text {ret ret }}$ mice showed the absence of immune cell infiltrates in the spinal cord parenchyma (SI Appendix, Fig. S1B).

Having established that the brain parenchyma of $P d g f b^{\text {rettret }}$ mice contains CD $45^{\text {hi }}$ cells, we used flow cytometry to identify immune cell populations. To characterize immune cell populations, leukocytes were isolated from the CNS and analyzed by flow cytometry. Flow cytometric analysis confirmed the immunohistochemistry findings and showed increased frequencies of CD45 hi cells in the CNS of pericyte-deficient mice. The majority of cells in the brains of $P d g f b^{\text {retret }}$ mice were $\mathrm{CD} 45^{\text {hi }} \mathrm{CD} 11 \mathrm{~b}^{+}$myeloid cells (mainly composed of Ly6C $\mathrm{C}^{\text {hi }}$ monocytes and CD11c ${ }^{+}$MHC-II high dendritic cells [DCs]) and $\mathrm{T}$ cells (Fig. 2E). Quantification of the absolute cell numbers of manually gated immune cell subsets in the brain showed a significant increase in the number of $\mathrm{CD} 45^{\text {hi }} \mathrm{CD} 11 \mathrm{~b}^{+}$myeloid cells, Ly6Chi MHC-II ${ }^{+}$monocyte-derived cells (MdCs), Ly6C ${ }^{\text {hi }}$ monocytes (Ly6G ${ }^{-} \mathrm{Ly}^{\mathrm{hi}} \mathrm{MHC}^{\mathrm{hi}} \mathrm{II}^{-}$myeloid cells), DCs (3.5\% of the $\mathrm{CD} 45^{\text {hi }} \mathrm{CD} 11 \mathrm{~b}^{+}$population), and $\mathrm{CD}^{+}$and $\mathrm{CD} 8^{+} \mathrm{T}$ cell populations compared with controls (Fig. $2 F$ ). Similarly to immunohistochemical analysis, no difference was detected in CD $45^{\text {hi }}$ cells in the spinal cord between control and $P d g f b^{\text {ret/ret }}$ mice using flow cytometry analysis (SI Appendix, Fig. S1 $C$ and $D$ ).

We next investigated the temporal aspect of leukocyte infiltration. ICAM-1 expression was detected by the developing vasculature in postnatal brains (postnatal day $[\mathrm{P}] 6, \mathrm{P} 16, \mathrm{P} 25$ ) in control and Pdgf $b^{\text {retret }}$ mice (SI Appendix, Fig. S3). We did not detect $\mathrm{CD} 45^{\mathrm{hi}}$ infiltrates in brain parenchyma at investigated postnatal stages $(\mathrm{P} 6, \mathrm{P} 16, \mathrm{P} 25)$ in $P d g f b^{\text {retret }}$ mice; however, frequent CD $45^{\mathrm{hi}}$ cells were detected inside the vessel lumen in 16- and 25-dold Pdgf $b^{\text {ret/ret }}$ mice (SI Appendix, Fig. S4). Thus, extravasation of leukocytes into brain parenchyma in $P d g f b^{\text {retret }}$ mice takes place after $\mathrm{P} 25$, a time point when the angiogenesis has gradually diminished and vasculature begins to stabilize (16).

Taken together, our data show that, in the absence of pericytes, the adult brain vasculature becomes permissive for leukocyte entry and that the infiltrated leukocyte population consists mostly of Ly6C ${ }^{\text {hi }}$ monocytes, MdCs, DCs, and T cells.

Characterization of Leukocyte Populations in Peripheral Organs in Pericyte-Deficient Adult Mice. We next analyzed leukocyte populations in blood as well as in primary and secondary lymphoid organs to ensure that the increased number of leukocytes in the brains of pericyte-deficient animals is not caused by peripheral alterations. The total cell number in thymus, spleen, axillary and inguinal lymph nodes, and blood was determined using an automated cell counter with isolated cells stained for further flow cytometry analysis. The cell number in the thymus, spleen, lymph node, and blood was comparable between $P d g f b^{\text {ret } / \text { ret }}$ and control mice (SI Appendix, Fig. S5A). Subsequent analysis of leukocyte populations did not show a skewing between $P d g f b^{\text {ret/ret }}$ and control mice in blood, lymph nodes, and spleen (SI Appendix, Fig. S5 B-D). There was no difference in the total leukocyte or neutrophil count in blood (SI Appendix, Fig. S5B), indicating the absence of systemic inflammation in Pdgf $b^{\text {retret }}$ mice. Histological examination of lymphoid organs did not show any differences in the spatial organization of T and B cells (SI Appendix, Fig. S5 $E-G$ ) between Pdgf $b^{\text {ret/ret }}$ and control mice.

We also investigated whether organs other than the brain presented with leukocyte infiltration or a spontaneous inflammation in $P d g f b^{\text {ret/ret }}$ mice. Flow cytometry analysis of immune cell populations was performed in the lung, liver, and small intestine of control and $P d g f b^{\text {ret/ret }}$ mice. No difference in any of the analyzed immune cell populations was observed between control and $P d g f b^{\text {rettret }}$ mice (SI Appendix, Fig. S6).

Thus, the increased number of infiltrated leukocyte subsets in the brain of adult pericyte-deficient mice is not due to increased numbers in the blood or peripheral organs.

Spatial Differences in Pericyte Coverage in the CNS of Pdgfb ${ }^{\text {ret/ret }}$ Mice. Previous studies have shown a negative correlation between pericyte coverage and BBB permeability in the brain (1, 2, 17-19). We therefore asked whether selective leukocyte infiltration into different CNS regions in $P d g f b^{\text {ret/ret }}$ mice can be explained by differences in capillary pericyte coverage in the brain and spinal cord. Therefore, we determined pericyte coverage in several other brain regions in addition to already reported areas (e.g., cortex and deep brain regions) $(1,17)$. Pericyte coverage in Pdgff $b^{\text {ret/ret }}$ mice was significantly reduced also in the cerebellum and brainstem $(\sim 42 \%$ and $\sim 54 \%$, respectively; SI Appendix, Fig. S7 $A$ and $B$ ). In agreement with reduced pericyte coverage, we observed a significantly increased VCAM-1 and ICAM-1 coverage in the brainstem and cerebellum of $P d g f b^{\text {retret }}$ mice (SI Appendix, Fig. S7 $C-F$ ) and numerous CD45 ${ }^{\text {hi }}$ cell infiltrates (SI Appendix, Fig. S7 $G-J$ ). Quantification of vessel surface pericyte coverage in the spinal cord showed that $P d g f b^{\text {ret/ret }}$ mice also have a significantly reduced capillary pericyte coverage compared with control animals in this CNS region (Fig. $3 A$ and $B$ ). However, the observed reduction ( 26\%) of pericyte coverage in the spinal cord vasculature of $P d g f b^{\text {retret }}$ mice is notably less than the previously reported reduction of pericyte coverage in the cortex or deep brain regions $(\sim 75 \%)$ $(1,17)$ or in the brainstem and cerebellum $(54 \%$ and $42 \%$, respectively; SI Appendix, Fig. S7 $A$ and $B$ ). In agreement with relatively complete pericyte coverage, the pattern and morphology of spinal cord vasculature of $P d g f b^{\text {retret }}$ mice appeared similar to control mice (Fig. $3 A$ ). The difference in vessel pericyte coverage between spinal cord and different brain regions in $P d g f b^{\text {ret } / r e t}$ mice indicates that spinal cord pericytes are less dependent on the platelet-derived growth factor (PDGF) B-PDGF receptor $\beta$ signaling axis for vascular recruitment.

Finally, we investigated whether higher capillary pericyte coverage in the spinal cord of $P d g f b^{\text {ret/ret }}$ mice parallels normalized expression of VCAM-1 and ICAM-1. Indeed, the expression of VCAM-1 and ICAM-1 on the spinal cord vasculature showed a similar zonal expression pattern in control and $P d g f b^{\text {ret/ret }}$ mice and only a slight increase in VCAM-1 and ICAM-1 coverage ( 3-4\%; Fig. $3 C-F)$.

Based on these data, we conclude that regional differences in the degree of capillary pericyte coverage in the CNS determine the extent to which the brain vasculature is permissive to leukocyte entry into the CNS under homeostasis. 
A Control $P d g f b^{\text {ret/ret }}$

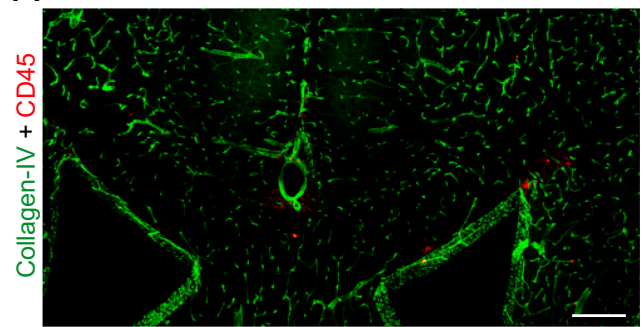

B Podocalyxin + CD45

Podocalyxin + CD45
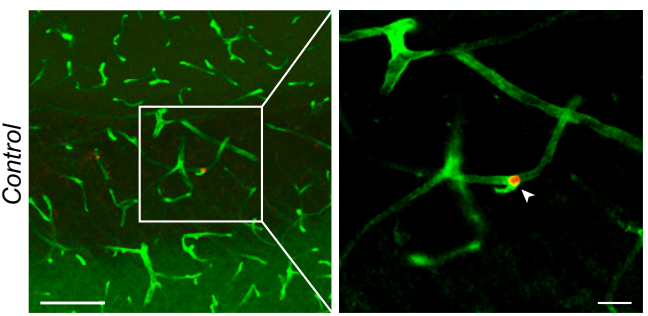

$\mathrm{CD} 45+\mathbb{D} \mathbb{A P}$

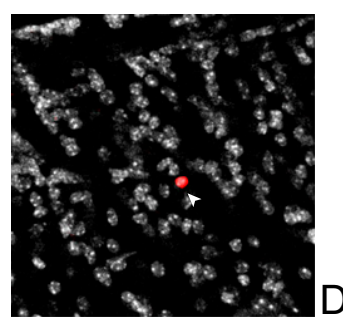

C
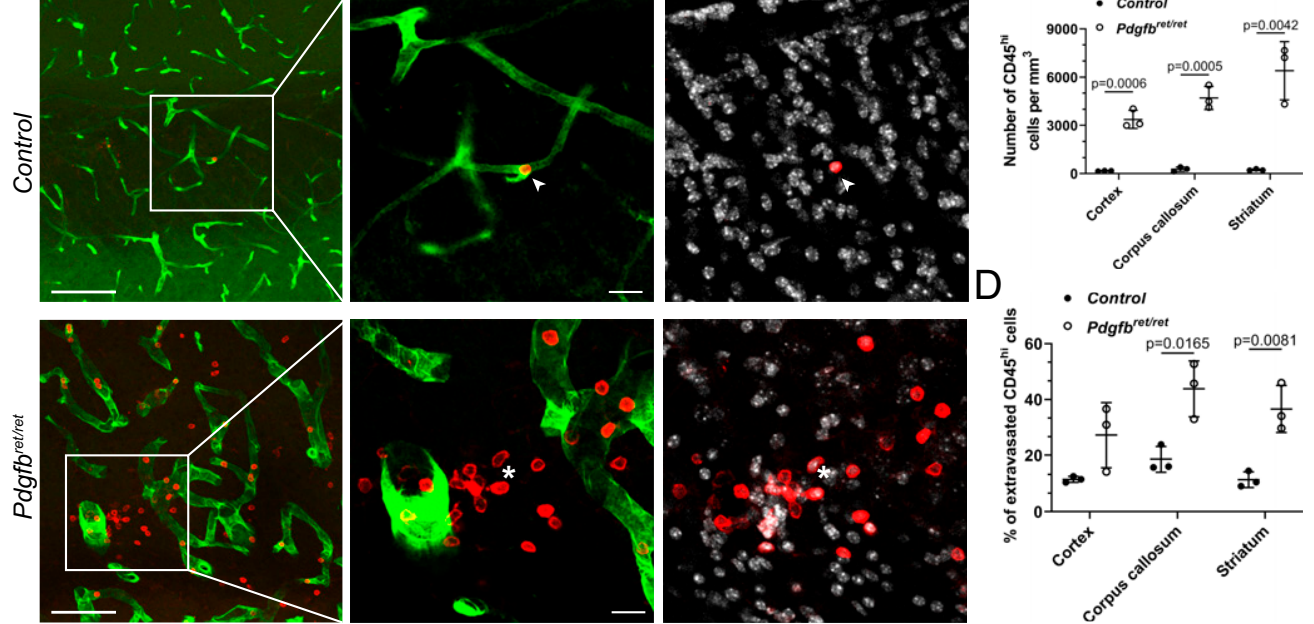

E

Control
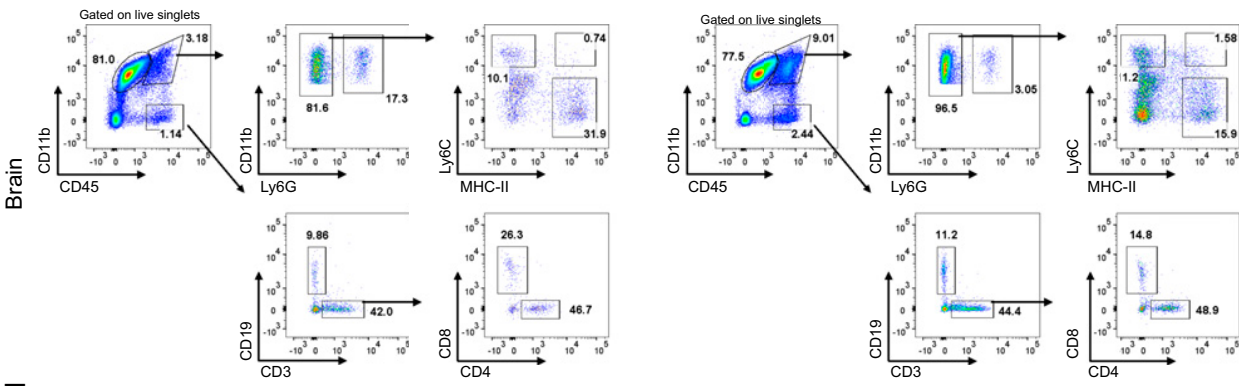

F

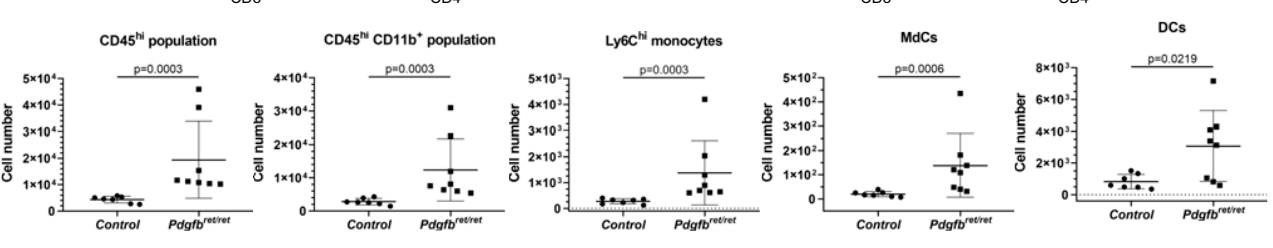

CD4* T cells

CD8* T cells

$B$ cells

Neutrophils
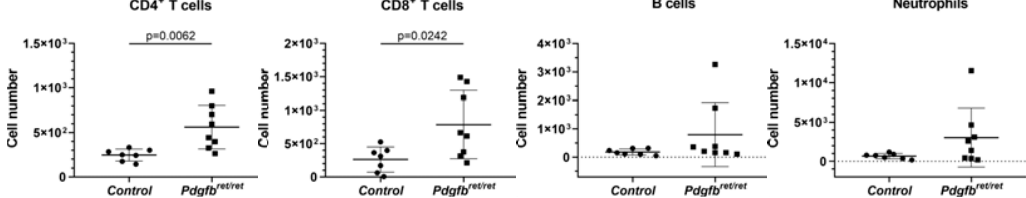

Fig. 2. Increased extravasation of leukocytes in the brain parenchyma of naïve pericyte-deficient brain. ( $A$ ) Overview images of the periventricular areas in the brains of control and pericyte-deficient mice $\left(P d g f^{\text {ret/ret }}\right)$. Arrowheads indicate leukocyte infiltrates (CD45, in red) in the parenchyma of $P d g f b^{\text {ret/ret }}$ mice. Blood vessels are visualized by collagen IV staining (in green). (B) High-magnification images showing a parenchymal infiltrate of CD45 ${ }^{\text {hi }}$ leukocytes (in red, asterisk) in the corpus callosum of Pdgfb ${ }^{\text {ret/ret }}$ mice. In control mice, few CD $45^{\text {hi }}$ leukocytes (arrowheads) are found only in the lumen of blood vessels (podocalyxin, in green). (C) Quantification of $C D 45^{\text {hi }}$ leukocytes in different anatomical regions in the brains of control and $P d g f b^{r e t / r e t}$ mice $(n=3)$. (D) Quantification of extravasated CD45 ${ }^{\text {hi }}$ leukocytes in different anatomical regions in the brains of control and Pdgfb ${ }^{\text {ret/ret }}$ mice $(n=3)$. One-way ANOVA followed by Tukey's post hoc test was used to determine the statistical significance. $(E$ ) Representative flow-cytometry pseudocolor plots showing the manual gating of microglia and other immune cell populations in the brain of naïve control and $P d g f b^{\text {ret/ret }}$ mice. $(F)$ Quantification of the absolute cell numbers of detected immune cell populations using flow cytometry in the brain ( $n=7-8$ mice per genotype). Pooled data from two independent experiments. Statistical significance was determined using unpaired $t$ test (DCs, CD 11 $\mathrm{C}^{+}, \mathrm{MHC}-\mathrm{II}{ }^{\text {high }}$, and CD4 $4^{+}$and CD8 ${ }^{+} \mathrm{T}$ cells) or Mann-Whitney $U$ test $\left(C D 45^{\text {hi }}\right.$ cells, CD45 ${ }^{\text {hi }}$ CD11 $\mathrm{b}^{+}$ cells, MdCs, Ly6Chi monocytes, neutrophils, and B cells). Data are presented as the mean \pm SD. (Scale bars: $A, 250 \mu \mathrm{m} ; B, 100 \mu \mathrm{m} ;$ Insets, $20 \mu \mathrm{m}$.) 

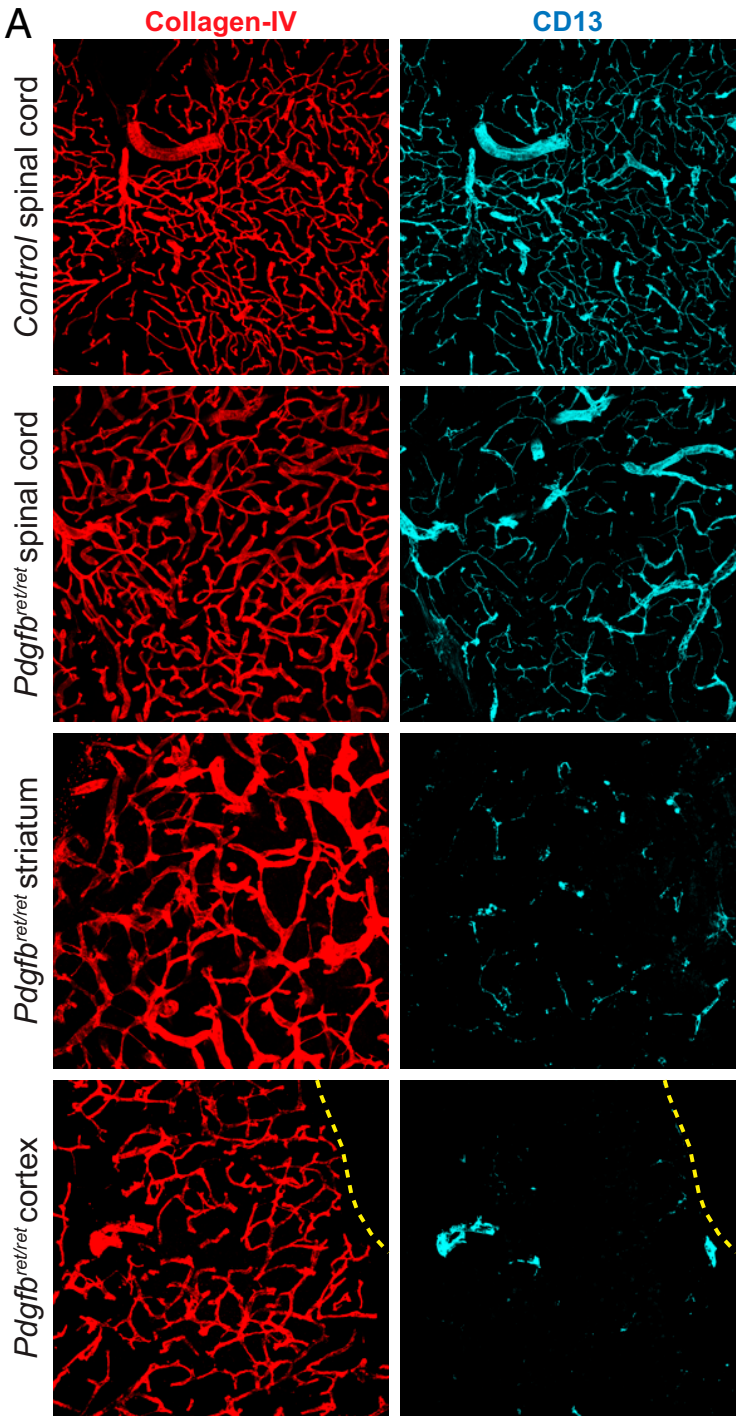

B

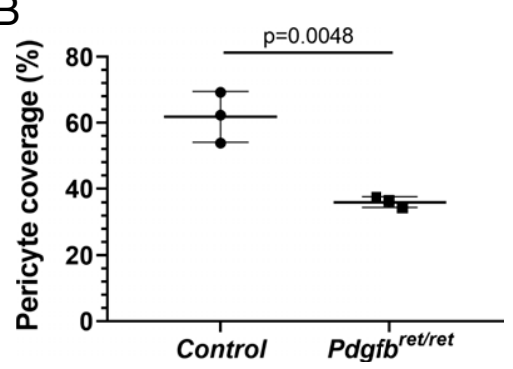

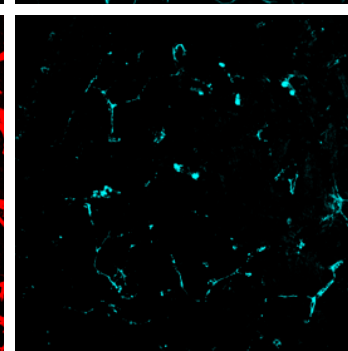

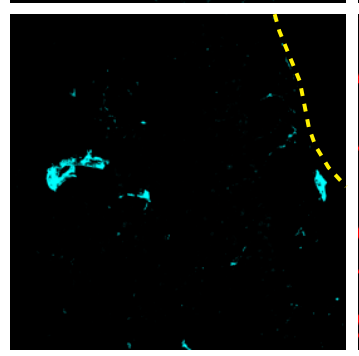

CD13
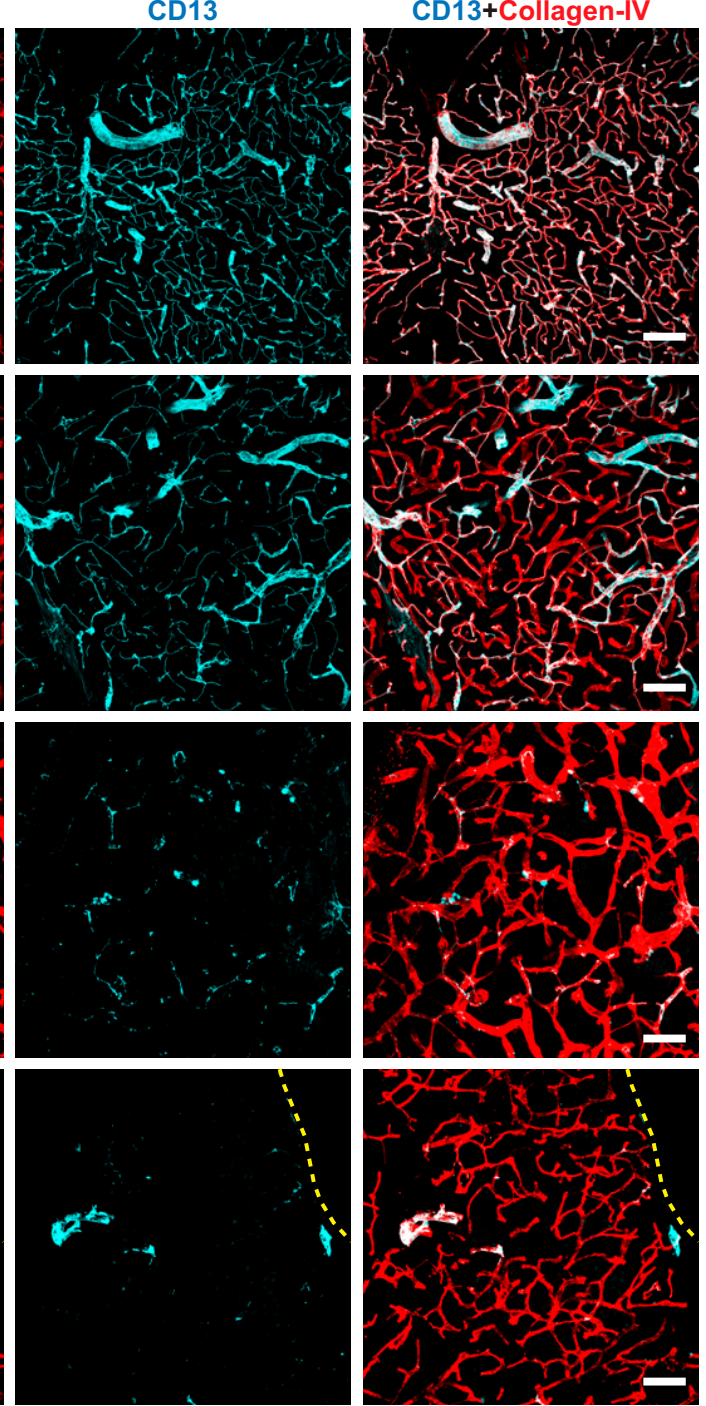

E

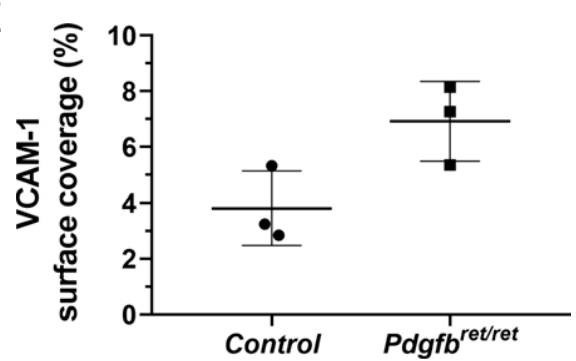

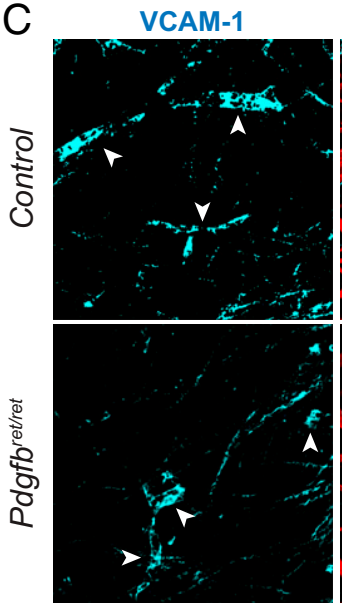

$\mathrm{D}$
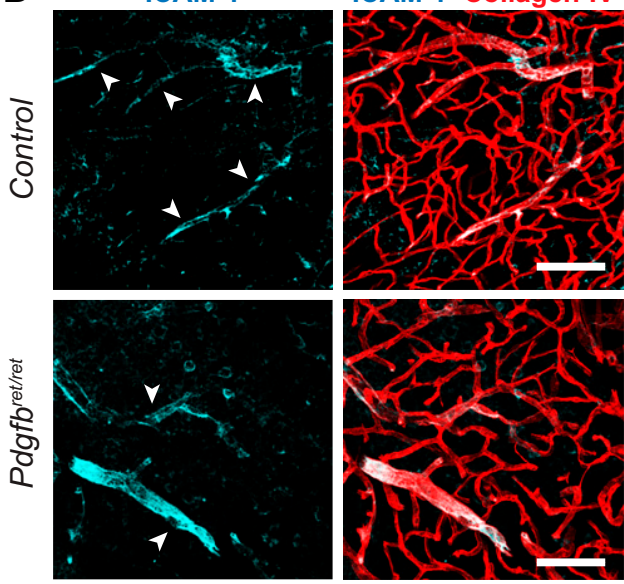

$\mathrm{F}$

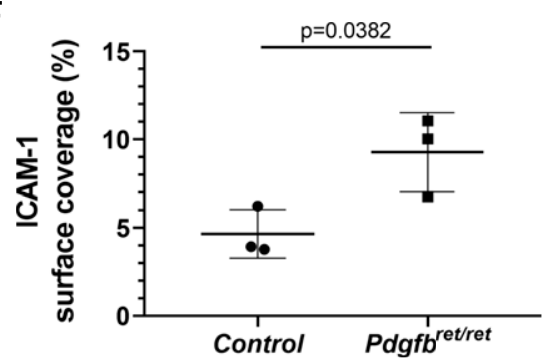

Fig. 3. Pericyte coverage and expression of LAMs in the spinal cord vessels of Pdgfb ${ }^{\text {retret }}$ mice. (A) Immunofluorescent staining of pericytes (CD13, in cyan) and vasculature (collagen IV, in red) in different anatomical regions of the CNS (spinal cord, striatum, cortex) in control and Pdgfb ${ }^{\text {retret }}$ mice. The yellow dotted line outlines the cortical surface. $(B)$ Quantification of vessel pericyte coverage in the spinal cord of control and Pdgfb ret/ret mice $(n=3$ mice per genotype). ( $C$ and $D$ ) VCAM-1 $(C)$ and ICAM-1 $(D)$ expression (in cyan) on the blood vessels (in red, collagen IV) in the spinal cord of control and Pdgfb ${ }^{\text {rettret }}$ mice. ( $E$ and $F$ ) Quantification of vascular surface coverage of VCAM-1 $(E)$ and ICAM-1 $(F)$ in the spinal cord of control and Pdgfb ${ }^{\text {rettret }}$ mice. (Scale bars: $100 \mu \mathrm{m}$.) Unpaired $t$ test was used to determine statistical significance.

Loss of Pericytes Does Not Alter Myelin Integrity. We next asked whether increased BBB permeability to plasma proteins $(1,2)$ and leukocytes leads to subclinical demyelination in the brains of pericyte-deficient mice. Myelin was visualized using Luxol fast blue-periodic acid Schiff (LFB-PAS) histochemical stains and immunofluorescent labeling with anti-myelin basic protein (MBP) antibody (SI Appendix, Fig. S8 $A$ and $B$ ). Quantification of LFBPAS and anti-MBP staining intensity in the region of high myelin content, the corpus callosum, did not differ between control and $P d g f b^{\text {rettret }}$ mice (SI Appendix, Fig. S8 $B-D$ ). A few brain sections of $P d g f b^{\text {ret/ret }}$ mice stained with LFB-PAS had a reduced staining intensity (score of 1 ) in the corpus callosum due to accompanying brain edema (1); however, demyelinating lesions were absent in the analyzed brain areas. Additionally, we did not detect differences in the ultrastructure of the myelin sheath between Pdgf $b^{\text {ret/ret }}$ and control mice (SI Appendix, Fig. S8E). 
Thus, infiltrated leukocytes in brain parenchyma in pericytedeficient mice do not initiate demyelinating pathology.

Pericyte-Deficient Mice Present an Aggravated, Atypical Experimental Autoimmune Encephalomyelitis Phenotype. We next investigated whether leukocyte-permissive vasculature modifies the course of autoimmune neuroinflammation. In order to address this question, we induced experimental autoimmune encephalomyelitis (EAE), an animal model of MS (20), in control and Pdgff $b^{\text {ret/ret }}$ mice. After active induction of EAE, which replicates both the induction and effector phase of the disease, $P d g f b^{\text {ret/ret }}$ mice presented with a severe, early-onset (4-5 d postimmunization) atypical phenotype as well as reduced survival (Fig. $4 A$ and $B$ and $S I$ Appendix, Fig. S9A and Table S2). We confirmed that control animals $\left(P d g f b^{w t / r e t}\right)$ did not differ from wild-type littermates in the clinical course of EAE (SI Appendix, Fig. S9 B and C). Therefore, $P d g f b^{w t / r e t}$ mice were continued to be used as controls. All control mice presented typical spinal cord EAE symptoms with ascending paralysis starting at the distal tail. All $P d g f b^{\text {ret/ret }}$ mice invariably developed atypical phenotype, which consisted of prominent cerebellar ataxia and spasticity, without ascending paralysis. To score the clinical severity of EAE in $P d g f b^{\text {ret/ret }}$ mice, we adopted an ataxia scoring protocol described by Guyenet et al. (21). We noticed that $P d g f b^{\text {retret }}$ mice presented with a basal ataxia score of 2 already at day 0 , which consisted of kyphosis (score of 1 ) and hindlimb clasping (score of 1 ; Fig. 4B; scoring protocol described in Materials and Methods). Adoptive transfer (passive) EAE resulted in the same aggravated atypical EAE in $P d g f b^{\text {retret }}$ mice as seen in active EAE (Fig. $4 C$ ), indicating that the severe phenotype is not due to a pathologically enhanced induction phase in pericyte-deficient mice. Immunization with a non-CNS antigen (ovalbumin peptide) using the same adjuvant did not result in clinical deficits in control or $P d g f b^{\text {ret/ret }}$ mice (SI Appendix, Fig. S9D).

We next investigated the spatial distribution of infiltrating cells in the CNS after induction of EAE using immunohistochemistry. This analysis showed an increased leukocyte infiltration into the brain parenchyma (cerebral cortex, striatum, corpus callosum, cerebellum, brainstem) of $P d g f b^{\text {ret/ret }}$ animals, whereas immune cell infiltrates were mostly found in the spinal cord in control animals (Fig. $4 D$ and $E$ and SI Appendix, Fig. S9 $E$ and $F$ ). The spinal cords of pericyte-deficient animals were devoid of $\mathrm{T}$ cell infiltrates, consistent with the atypical clinical phenotype (Fig. $4 E$ ) and with relatively complete vessel pericyte coverage (Fig. 3). Assessment of myelin damage after the induction of EAE showed pronounced demyelination in the brains of pericyte-deficient mice compared with control animals (SI Appendix, Fig. S9 $G$ and $H$ ).

We next analyzed which immune cells infiltrate the CNS after active immunization in control and $P d g f b^{\text {ret/ret }}$ mice. Flow cytometry analysis confirmed the immunohistochemistry results showing an increased number of CD45 ${ }^{\text {hi }}$ leukocytes in the brains in $P d g f b^{\text {ret/ret }}$ mice compared with controls (Fig. $4 F$ and SI Appendix, Fig. S10A). The majority of these infiltrates (approximately $60 \%$ of live singlets) in Pdgf $b^{\text {ret/ret }}$ mice were CD $45^{\mathrm{hi}} \mathrm{CD} 11 \mathrm{~b}^{+}$myeloid cells. Within this population, we detected a significant increase of $\mathrm{Ly}^{6} \mathrm{C}^{\mathrm{hi}}$ monocytes and $\mathrm{MdC}$ subpopulations in the brains of $P d g f b^{\text {ret/ret }}$ mice compared with controls but no differences in neutrophil or B cell numbers (Fig. $4 F$ and SI Appendix, Fig. S9I). In agreement with the clinical deficits and immunohistochemistry, the spinal cord of $P d g f b^{\text {ret/ret }}$ mice was essentially devoid of leukocytes compared with controls (Fig. $4 G$ and SI Appendix, Figs. S9J and S10B).

Thus, $P d g f b^{\text {ret } / r e t}$ mice develop a severe, atypical EAE phenotype and show spatially restricted infiltration of inflammatory cells predominantly into the brain, consisting mostly of Ly6 $\mathrm{C}^{\text {hi }}$ monocytes and $\mathrm{MdC}$ populations.
MS Drug Fingolimod (FTY-720) Ameliorates the Severe Atypical EAE Phenotype of $\boldsymbol{P d g} \boldsymbol{f b}^{\text {ret/ret }}$ Mice. We next addressed whether the aggravated phenotype of $P d g f b^{\text {ret/ret }}$ mice after induction of EAE is caused by the massive influx of peripheral immune cells into the CNS. Mice were treated daily, starting on day 4 postimmunization, with FTY-720 (fingolimod), a functional antagonist of sphingosine1-phosphate receptor 1 (S1P1), which causes leukopenia by blocking the egress of lymphocytes from lymph nodes (22). All vehicle-treated $P d g f b^{\text {retret }}$ mice reached termination criteria (ataxia score of $8.5-10$ ) after EAE induction, whereas FTY-720-treated $P d g f b^{\text {ret } / r e t}$ mice did not develop symptoms of EAE during the course of the experiment ( $25 \mathrm{~d}$; Fig. $5 A$ and $B$ ). Of note, the ataxia score of 2 observed in all $P d g f b^{\text {ret } / \text { ret }}$ mice before FTY-720 administration was not alleviated by FTY-720 treatment. As expected, the EAE score of vehicle-treated control animals improved by day 25 postimmunization. In addition, FTY-720-treated control mice did not develop EAE (Fig. 5B and SI Appendix, Fig. S11A). Flow cytometry analysis of peripheral blood on day 12 postimmunization with MOG peptide confirmed the FTY-720 treatment-induced leukopenia in control and $P d g f b^{\text {ret/ret }}$ mice (SI Appendix, Fig. S11 B-E). Flow cytometric analysis of immune cells in the brains and spinal cords of vehicle- and FTY720 -treated $P d g f b^{\text {ret } / \text { ret }}$ mice was performed on the same day when vehicle-treated mice reached termination criteria (ataxia score of 8.5-10). In parallel, brains and spinal cords of vehicle- and FTY720-treated control mice were analyzed on the same day when vehicle-treated control mice reached EAE score of 3-3.5. As expected, FTY-720-treated animals had significantly lower numbers of CD45 $5^{\mathrm{hi}}$ immune cells in the CNS both in control and $P d g f b^{\text {ret/ret }}$ mice (Fig. $5 C$ and $D$ and SI Appendix, Fig. S11 $F$ and $G)$. In addition to reduced number of $\mathrm{CD} 4^{+}$and $\mathrm{CD} 8^{+} \mathrm{T}$ cells, we also observed a reduction of myeloid cells $\left(\mathrm{Ly} 6 \mathrm{C}^{\text {hi }}\right.$ monocytes and MdCs) after FTY-720 treatment in the brains of $P d g f b^{\text {ret ret }}$ mice. FTY-720 treatment after the induction of EAE has been shown to reduce the number of circulating monocytes in addition to $\mathrm{T}$ cells (23), which could explain the significantly reduced myeloid cells in the spinal cord of control mice as well as in the brains of both control and $P d g f b^{\text {rettret }}$ mice (Fig. $5 C$ and $D$ and SI Appendix, Fig. S11 $F$ and $G$ ). Immunohistochemical analysis confirmed the reduced infiltration of leukocytes into the brain of pericyte-deficient mice after FTY-720 treatment (SI Appendix, Fig. S11H).

Thus, we conclude that the mortality of pericyte-deficient mice after induction of EAE is caused by excessive entry of peripheral immune cells into the brain and neuroinflammation.

\section{Combined Anti-VCAM-1 and Anti-ICAM-1 Treatment Mitigates the Severe} Atypical EAE Phenotype of $\boldsymbol{P d g} \boldsymbol{f b}^{\text {ret/ret }}$ Mice. Pericyte deficiency alters endothelial cell phenotype and leads to up-regulation of LAMs on the brain endothelium, which are important for leukocyte transmigration. We therefore addressed whether the massive infiltration of leukocytes can be modulated by blocking ICAM-1 and VCAM-1 after the induction of EAE, and whether this will alter the course of the disease. Mice were treated daily with a mix of anti-VCAM-1 and anti-ICAM-1 monoclonal antibodies starting on day 1 postimmunization. Control mice treated with isotype control reached termination criteria (EAE score of 3-3.5), while control mice treated with anti-VCAM-1 and anti-ICAM-1 antibodies developed no symptoms or milder symptoms that stagnated (Fig. 6A). All IgG isotype control-treated $P d g f b^{\text {ret/ret }}$ mice reached termination criteria similarly to previous experiments, whereas anti-VCAM-1 and anti-ICAM-1 treatment ameliorated the ataxia symptoms and rescued the mortality of $P d g f b^{\text {rettret }}$ mice (Fig. $6 B$ and $C$ ). In agreement with this improved phenotype, flow cytometric analysis showed a significant reduction in the total number of $\mathrm{CD} 45^{\mathrm{hi}}$ leukocytes in the brains of $P d g f b^{\text {retret }}$ mice and in the spinal cords of control mice treated with anti-VCAM-1 and anti-ICAM-1 antibodies compared with isotype control-treated mice (Fig. $6 D$ and $E$ and SI Appendix, Fig. S12). 
A

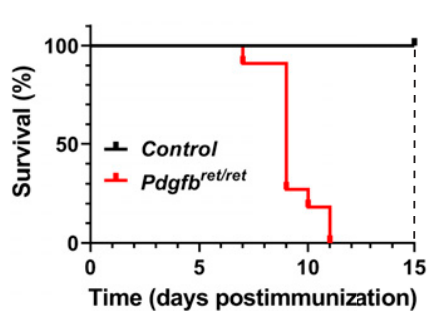

D

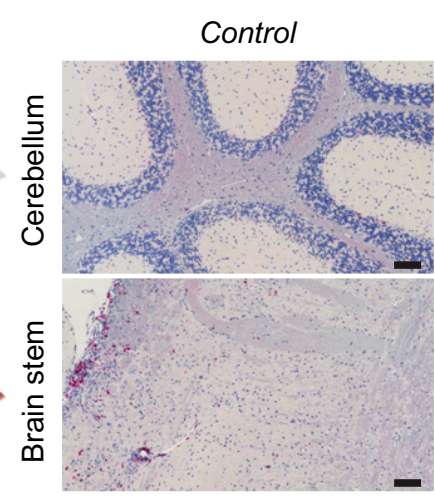

B

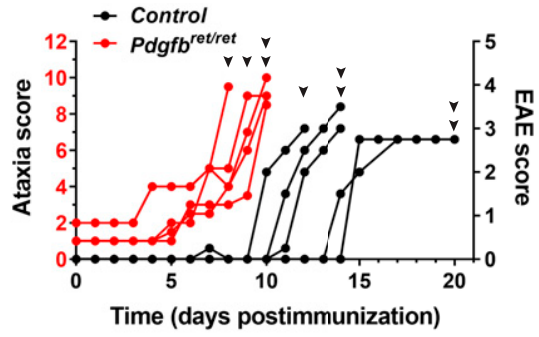

C

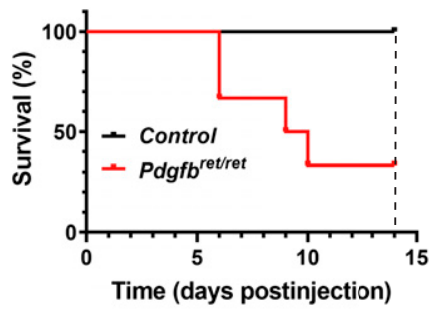

Pdgfb retret

E

Control

$P d g f b^{\text {retret }}$
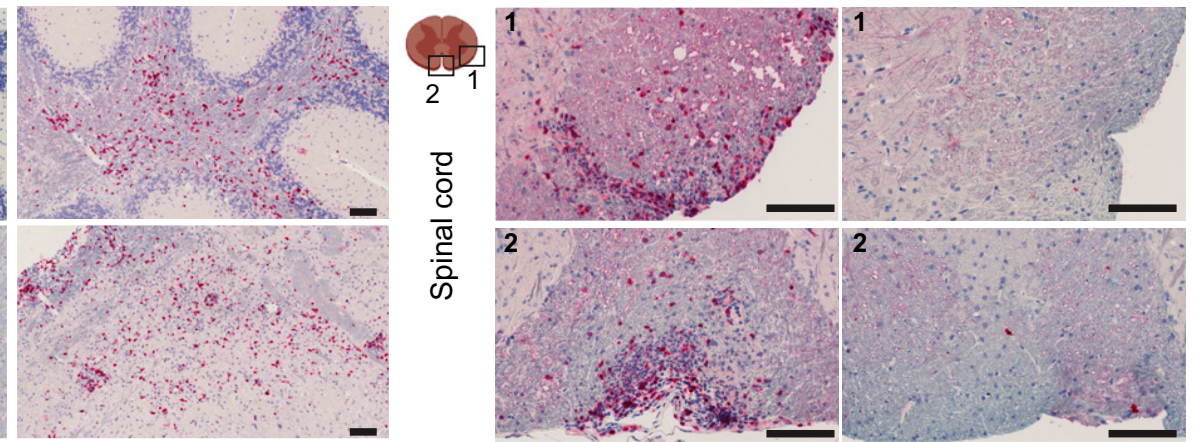

$\mathrm{CD4}^{+} \mathrm{T}$ cells

$\mathrm{CD}^{+} \mathrm{T}$ cells
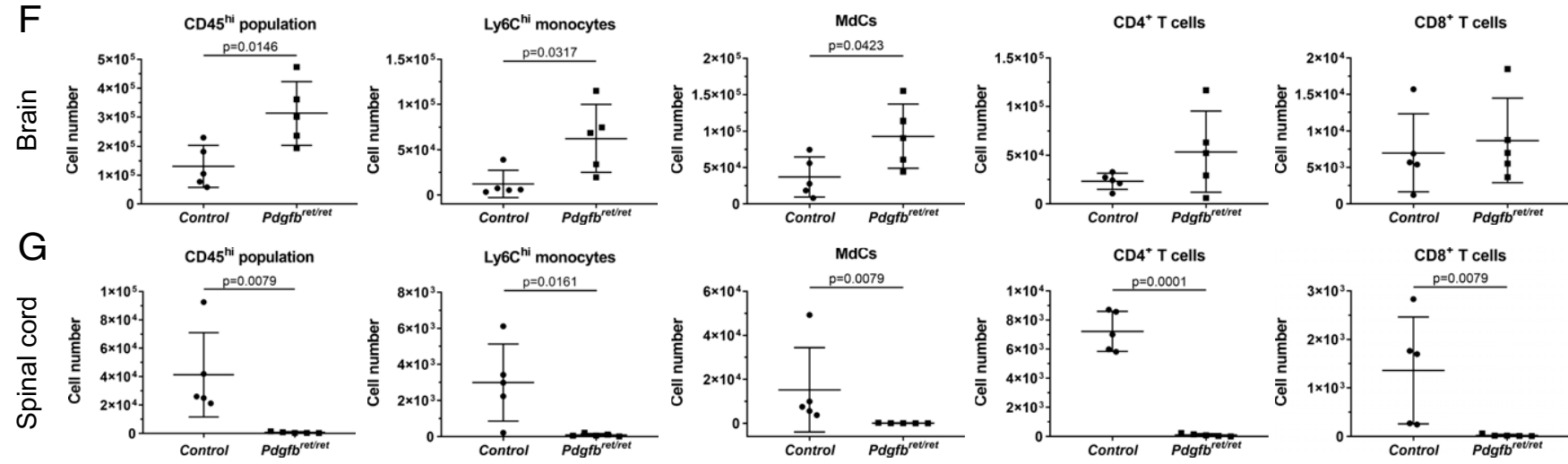

Fig. 4. Pericyte-deficient mice die with atypical EAE accompanied by increased leukocyte infiltration into the brain. (A) Kaplan-Meier survival curves after active induction of EAE. The experiment was terminated on day 15, indicated by black dashed line. Pooled data from two individual experiments $(n=11$ mice per genotype). Survival curves showed statistical difference $(P<0.0001$, log-rank test). (B) Scoring of neurological symptoms during the course of active EAE. The left $y$ axis shows cerebellar ataxia scores of $P d g f b^{\text {ret }}{ }^{\text {ret }}$ mice (in red), and the right $y$ axis shows classical EAE scores of control mice (in black). Arrowheads indicate when individual mice were euthanized for flow-cytometry analysis. Materials and Methods includes detailed termination criteria. Each line represents symptoms of an individual mouse ( $n=5$ mice per genotype, showing two pooled experiments). (C) Kaplan-Meier survival curves after passive induction of EAE. The experiment was terminated on day 14, indicated by black dashed line (controls, $n=5 ;$ Pdgfb ${ }^{\text {ret/ret }}, n=6$ ). Survival curves showed a statistically significant difference $(P=0.0300$, log-rank test). $(D)$ Immunohistochemical staining of T cells (CD3, in red) of sagittal brain sections of the cerebellum and brainstem after active induction of EAE of control (on day 16 postimmunization) and Pdgfb ${ }^{\text {retret }}$ mice (on day 11 postimmunization). (E) Immunohistochemical staining of $\mathrm{T}$ cells $(C D 3$, in red) on coronal sections of the spinal cords showing two regions $(1,2)$ after active induction of EAE in control (on day 16 postimmunization) and $P d g f b^{r e t / r e t}$ mice (on day 11 postimmunization). Tissue sections were counterstained with hematoxylin $(D$ and $E)$. $(F$ and $G)$ Quantification of the absolute cell numbers of different leukocyte populations (gated as shown in SI Appendix, Fig. S10) in the brains (cerebrum, cerebellum, and brainstem; $F$ ) and spinal cords (G) of control (EAE score of 3-3.5) and Pdgfb ${ }^{\text {ret/ret }}$ (ataxia score, $n=9-10$ ) mice using flow cytometry. Shown are pooled data from two individual experiments ( $n=5$ mice per genotype). Data are presented as the mean \pm SD. Statistical significance was determined using unpaired $t$ test (brain, CD45 hi, MdCs, and CD4 ${ }^{+} \mathrm{T}$ and CD8 ${ }^{+} \mathrm{T}$ cells; spinal cord, CD4 ${ }^{+} \mathrm{T}$ and Ly6 $\mathrm{C}^{\text {hi }}$ monocytes) or Mann-Whitney $U$ test (brain, Ly6C ${ }^{\text {hi }}$ monocytes; spinal cord, CD45 hi, CD8 ${ }^{+} \mathrm{T}$ cells, and MdCs). (Scale bars: $D$ and $E, 100 \mu \mathrm{m}$.)

Thus, blocking LAMs on the endothelium alleviated the severe clinical phenotype of pericyte-deficient mice, indicating that the proinflammatory phenotype of the endothelium caused by pericyte deficiency contributes to the aggravated leukocyte extravasation during neuroinflammation.

\section{Spontaneous Neuroinflammation in Pdgfb ${ }^{\text {ret/ret }}$ Mice Expressing} Myelin-Specific T Cell Receptor. Little is known about what triggers spontaneous activation and entry of self-reactive $\mathrm{T}$ cells into the CNS. We asked whether the leukocyte-permissive NVU in pericytedeficient animals leads to spontaneous neuroinflammation when there is an overabundance of self-reactive $\mathrm{T}$ cells toward a myelin antigen. To answer this question, we crossed $P d g f b^{\text {retret }}$ mice with 2D2 mice, which express a $\mathrm{MOG}_{35-55}$ peptide-specific $\mathrm{T}$ cell receptor (TCR) (24). Previous studies have reported that $\sim 5 \%$ of $2 \mathrm{D} 2$ mice develop spontaneous EAE with classical symptoms (24). Offspring of $P d g f b^{\text {retret }}$ and $P d g f b^{\text {wtret }} ; 2 D 2^{\text {tg }}$ crosses were monitored after weaning for signs of cerebellar ataxia and classical EAE. Consistent with previous observations (Figs. $4 B, 5 B$, and $6 C$ ), all animals carrying two alleles of mutated $P d g f b\left(P d g f b^{\text {retret }}\right)$ presented with an ataxia score of 2 , consisting of hindlimb clasping (score of 1) and kyphosis (score of 1) already at weaning, which remained stable (Fig. $7 A$ ). However, 
A

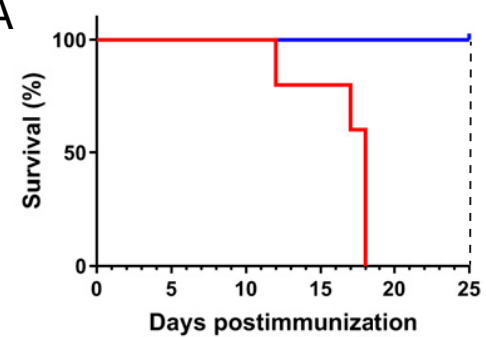

C
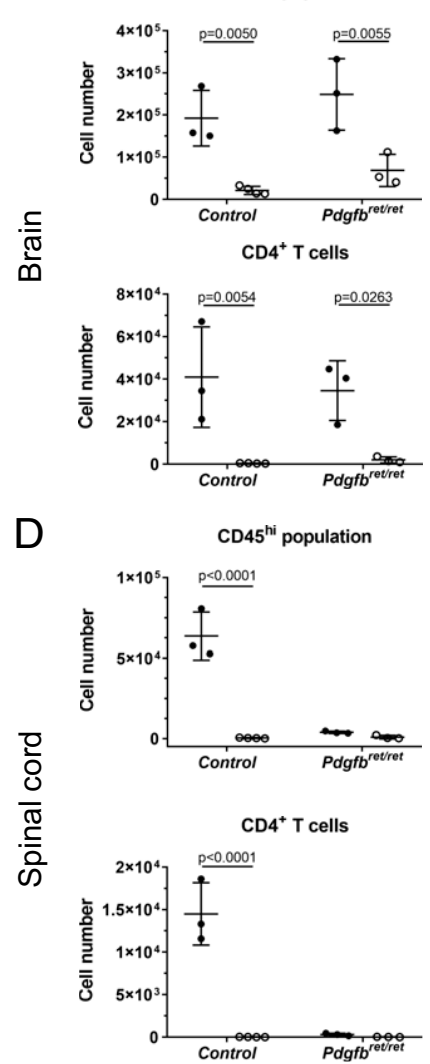

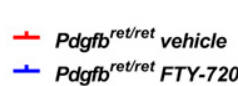

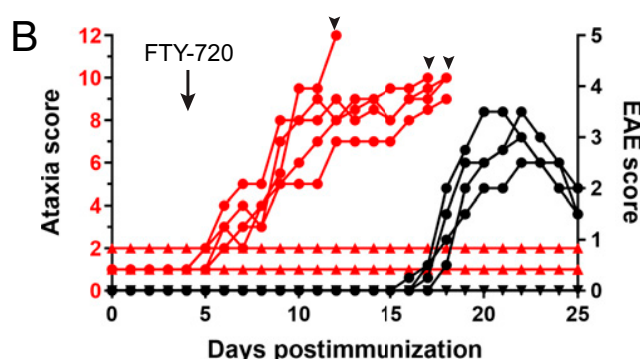

Days postimmunization
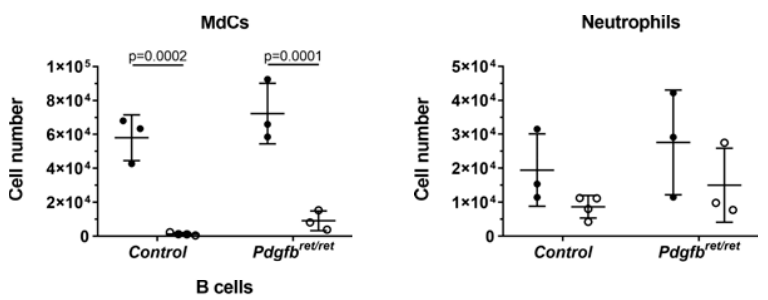

$\mathrm{CD}^{+} \mathrm{T}$ cells
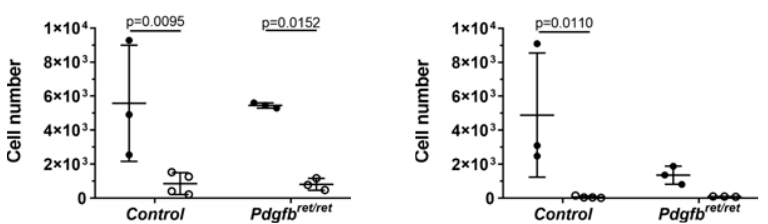

- Vehicle

- FTY-720

Ly6C $^{\text {hi }}$ monocytes
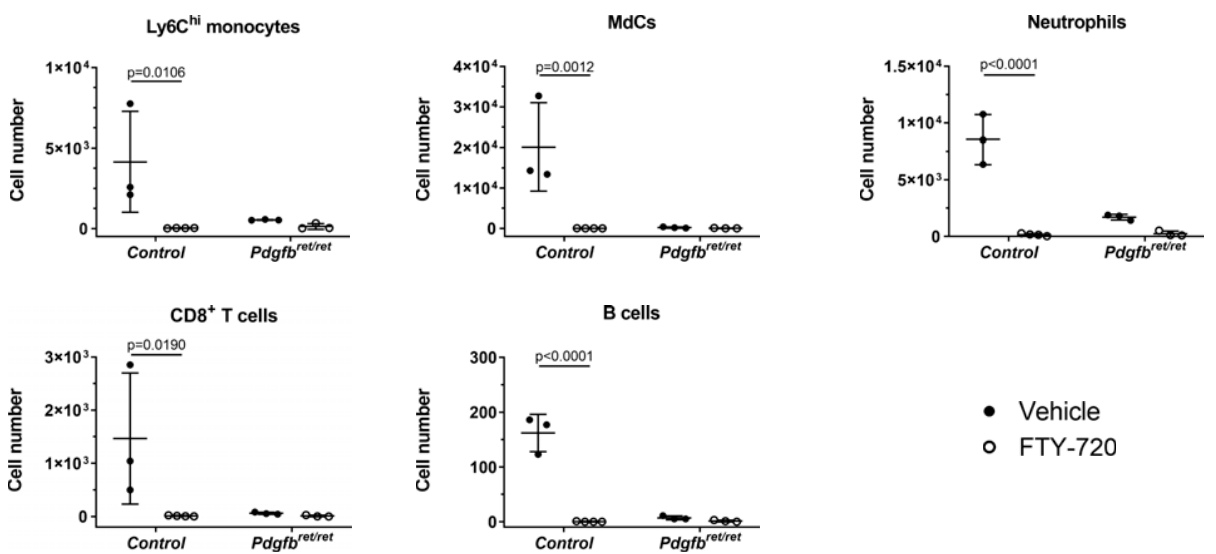

$\rightarrow-$ Control vehicle $(n=4)$

- Pdgfb ${ }^{\text {retret }}$ vehicle ( $\left.n=5\right)$

- Control FTY-720 ( $n=6)$

- Pdgfb ${ }^{\text {retret }}$ FTY-720 (n=6)

Fig. 5. FTY-720 treatment rescues the lethality of pericyte-deficient mice after induction of EAE. ( $A$ ) Kaplan-Meier survival curves of vehicle and FTY720-treated $P d g f^{\text {ret/ret }}$ mice after active induction of EAE $(P=0.002$, log-rank test). The experiment was terminated on day 25 (marked by a black dashed line; $n=5$ mice treated with vehicle, $n=6$ mice treated with FTY-720). (B) Scoring of clinical symptoms during the course of FTY-720 treatment after induction of active EAE of control and $P d g f^{\text {ret/ret }}$ mice. The left $y$ axis shows the cerebellar ataxia scores of $P d g f^{\text {ret/ret }}$ mice (in red) and the right $y$ axis the classical EAE scores of control mice (in black). FTY-720 administration $(0.5 \mathrm{mg} / \mathrm{kg}$ ) was started on day 4 postimmunization (black arrow), and the experiment was terminated on day 25 postimmunization. The ataxia score or EAE score of each mouse is plotted individually. Arrowheads indicate when Pdgfb ${ }^{\text {ret/ret }}$ mice reached termination criteria and were euthanized ( $n=4-5$ mice per group). ( $C$ and $D$ ) Quantification of the absolute cell numbers of the different immune cell populations (gating shown in SI Appendix, Fig. S11 F and G) in the brain (C) and spinal cords (D) of vehicle- and FTY-720-treated control and Pdgfb ${ }^{\text {ret/ret }}$ mice using flow cytometry. Controls, $n=3$ vehicle-treated and $n=4$ FTY-720-treated; Pdgfb ${ }^{\text {retret }}$ mice, $n=3$ vehicle-treated and $n=3$ FTY-720-treated. Pooled data from two independent experiments. Data are presented as the mean \pm SD. Two-way ANOVA followed by Šídák's post hoc test was used to determine statistical significance between groups.

$P d g f b^{\text {retret }} ; 2 D 2^{\text {tg }}$ mice showed increasing cerebellar ataxia scores compared with $P d g f b^{\text {rettret }} ; 2 D 2^{\text {neg }}$ mice (Fig. $7 A$ ). Other control mice $\left(P d g f b^{\text {wt/ret }} ; 2 D 2^{\text {neg }}, P d g f b^{\text {wt/ret }} ; 2 D 2^{\text {tg }}\right)$ occasionally received a score of 1 , which was based on single balance loss on the ledge test. Of note, the ataxia scores of individual $P d g f b^{\text {ret/ret }} ; 2 D 2^{\text {tg }}$ mice fluctuated over the monitored time period. None of the mice developed signs of classical EAE. Immunofluorescent staining of the brains of 3-mo-old $P d g f b^{\text {rettret }} ; 2 D 2^{\text {tg }}$ animals showed an increased number of $\mathrm{CD} 45^{\text {hi }}$-positive cells in the brains compared with $P d g f b^{\text {ret } / \text { ret }} ; 2 D 2^{\text {neg }}$ mice (Fig. $7 B$ ). In support of this finding, flow cytometric analysis confirmed that the brains of $P d g f b^{\text {retrret }} ; 2 D 2^{t g}$ mice contained a significantly higher number of CD45 $5^{\text {hi }}$ cells compared with $P d g f b^{\text {retret }} ; 2 D 2^{\text {neg }}$ mice (Fig. $7 C$ and SI Appendix, Fig. S13A).
$P d g f b^{\text {retret }} ; 2 D 2^{\text {tg }}$ animals and all control animals were euthanized for flow cytometry when $P d g f b^{\text {retret }} ; 2 D 2^{\text {tg }}$ animals had reached the ataxia score of 6-9. Immune cell infiltrates in the brains of $P d g f b^{\text {retrret }}$ and $P d g f b^{\text {retret }} ; 2 D 2^{\text {tg }}$ mice consisted of Ly6C $\mathrm{C}^{\text {hi }}$ monocytes, MdCs, and $\mathrm{CD}^{+}$and $\mathrm{CD}^{+} \mathrm{T}$ cells (Fig. $7 C$ ). Interestingly, in $P d g f b^{\text {rettret }} ; 2 \mathrm{D} 2^{\text {tg }}$ mice, in addition to the aforementioned populations, neutrophils and B cells were also detected. Furthermore, spinal cords of $P d g f b^{\text {rettret }} ; 2 D 2^{\text {tg }}$ mice showed a significant increase in immune cell infiltrates; however, the total cell numbers were low compared with brains (SI Appendix, Fig. S13 B and $C$ ).

Thus, these experiments demonstrate that the leukocytepermissive NVU caused by reduced pericyte coverage promotes 

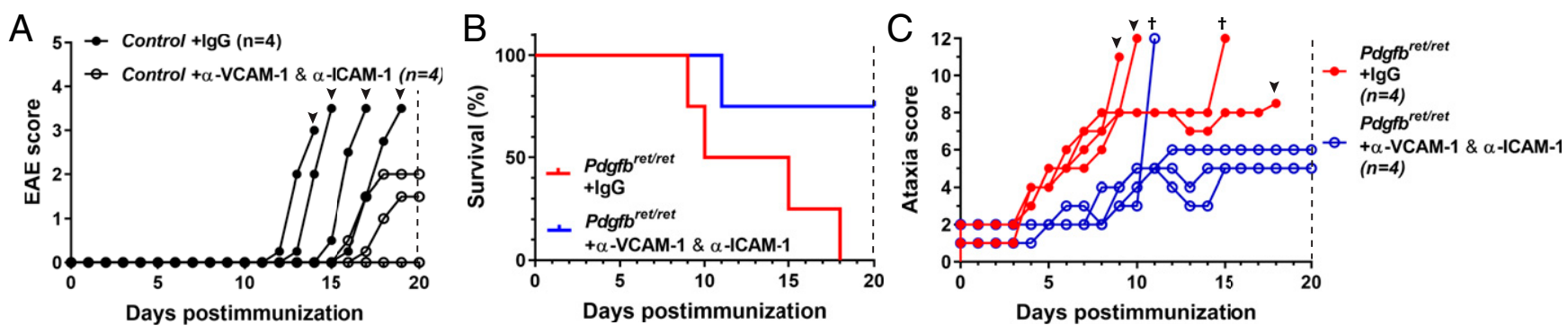

D
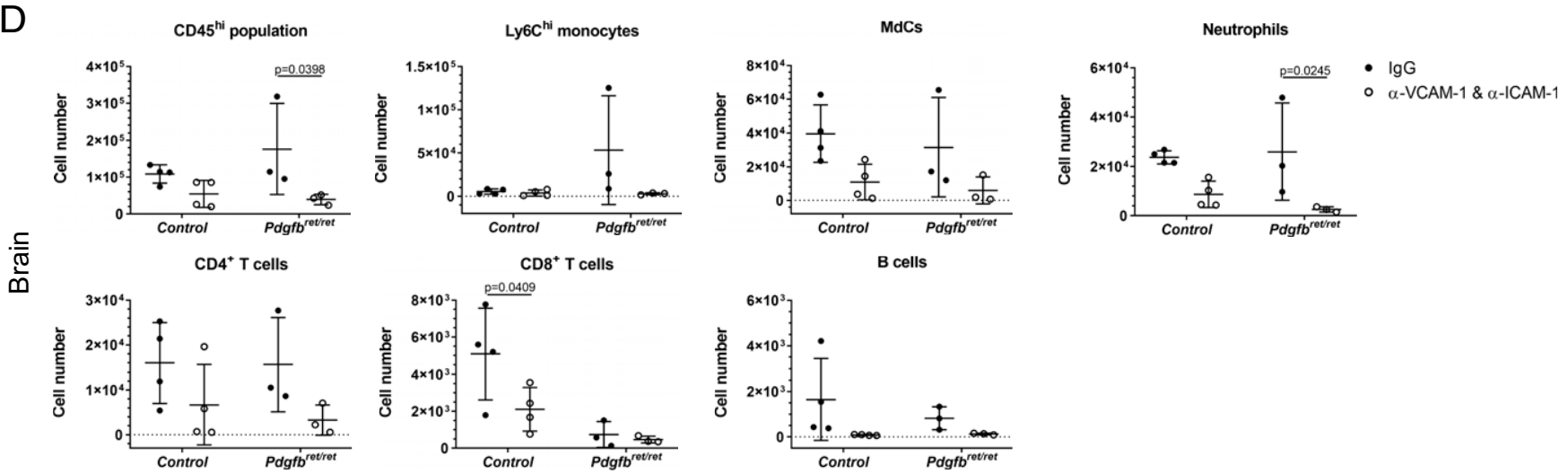

E
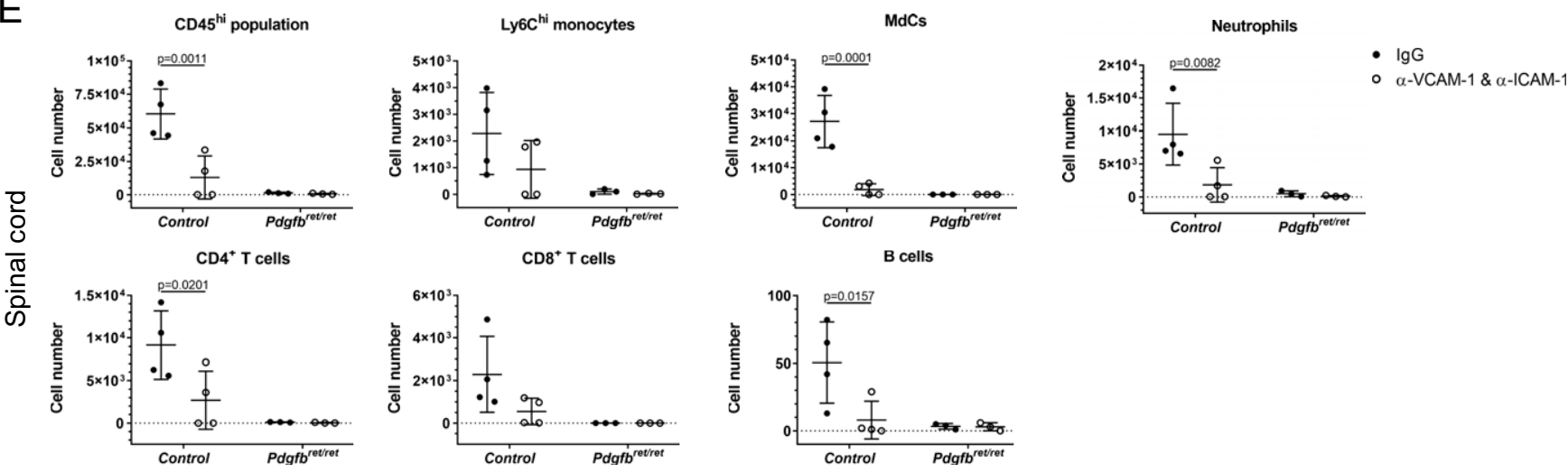

Fig. 6. Combined treatment using anti-VCAM-1 and anti-ICAM-1 antibodies ameliorates symptoms of pericyte-deficient and control mice after the induction of EAE. (A) Scoring of clinical symptoms during the course of anti-VCAM-1 and anti-ICAM-1 mAb treatment after induction of active EAE of control mice. (B) Kaplan-Meier survival curves of isotype control and anti-VCAM-1 and anti-ICAM-1 monoclonal antibody ( $\alpha$-VCAM-1 and $\alpha-I C A M-1$ mAb) treated $P d g f^{\text {retret }}$ mice after active induction of EAE $(P=0.05$, log-rank test). (C) Scoring of clinical symptoms during the course of anti-VCAM-1 and anti-ICAM-1 mAb treatment after induction of active EAE in Pdgfb ${ }^{\text {retret }}$ mice $(C)$. (A-C) The EAE or ataxia score of each mouse is plotted. Arrowheads indicate when mice reached termination criteria and were euthanized for analysis. The experiment was terminated on day 20 (black dashed line; $n=4$ mice treated with isotype control, $n=4$ mice treated with anti-VCAM-1 and anti-ICAM-1 mAb). ( $D$ and $E$ ) Quantification of the absolute cell numbers of different immune cell populations (gating shown in SI Appendix, Fig. $S 12$ ) in the brains $(D)$ and spinal cords $(E)$ of isotype control and anti-VCAM-1 and anti-ICAM-1 mAb-treated control and Pdgfb $b^{\text {retret }}$ mice using flow cytometry. Controls, $n=4$ mice per group; Pdgfb ${ }^{\text {retret }}$ mice, $n=3$ mice per group. Pooled data from two independent experiments. Data are presented as the mean \pm SD. Two-way ANOVA followed by Š́dák's post hoc test was used to determine statistical significance between groups.

the development of a neuroinflammatory disorder associated with increased myelin-reactive $\mathrm{T}$ cells in the circulation.

\section{Discussion}

Pericytes have been shown to regulate BBB integrity at the level of endothelial transcytosis $(1,2)$. Pericytes also induce polarization of astrocyte end-feet (1); however, the extent of pericyte control over other characteristics of the brain vasculature is less explored. In this study, we investigated the role of pericytes in regulating leukocyte trafficking into the adult CNS. In addition, we show that, in the absence of pericytes, the NVU becomes permissive to leukocyte entry, leading to aggravated neuroinflammation in a setting of autoimmunity.
A previous study on pericyte-deficient $P d g f b^{-/-}$embryos showed that several LAMs (e.g., Icam1, Alcam, Lgals3) were significantly up-regulated on the brain vasculature (2). In addition, a modest increase in Ly-6G/Ly-6C-positive leukocytes was observed in the brains of juvenile $P d g f b^{F 7 / F 7}$ mice that display a $50 \%$ reduction in pericyte coverage compared with controls (2). Our observation that several LAMs, including VCAM-1 and ICAM-1, are upregulated in the adult vasculature of $P d g f f^{\text {ret ret }}$ mice, which is accompanied by increased leukocyte entry into the brain parenchyma, corroborates and extends these findings. The up-regulation of VCAM-1 and ICAM-1 are important steps of the cascade of leukocyte transmigration (reviewed in ref. 14). Accordingly, 
A

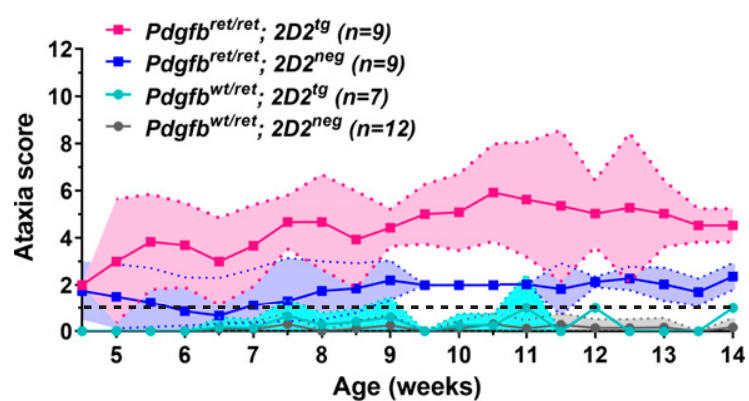

C
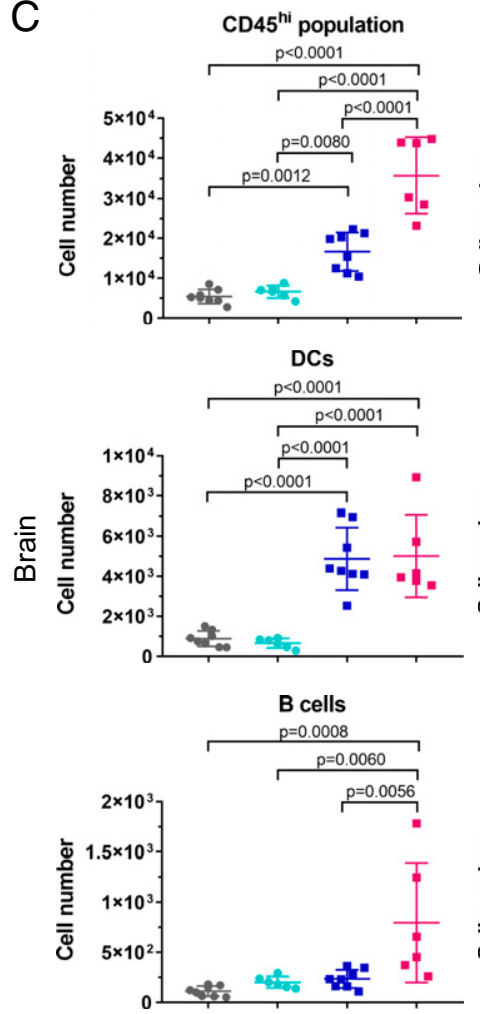

$\mathrm{B}$
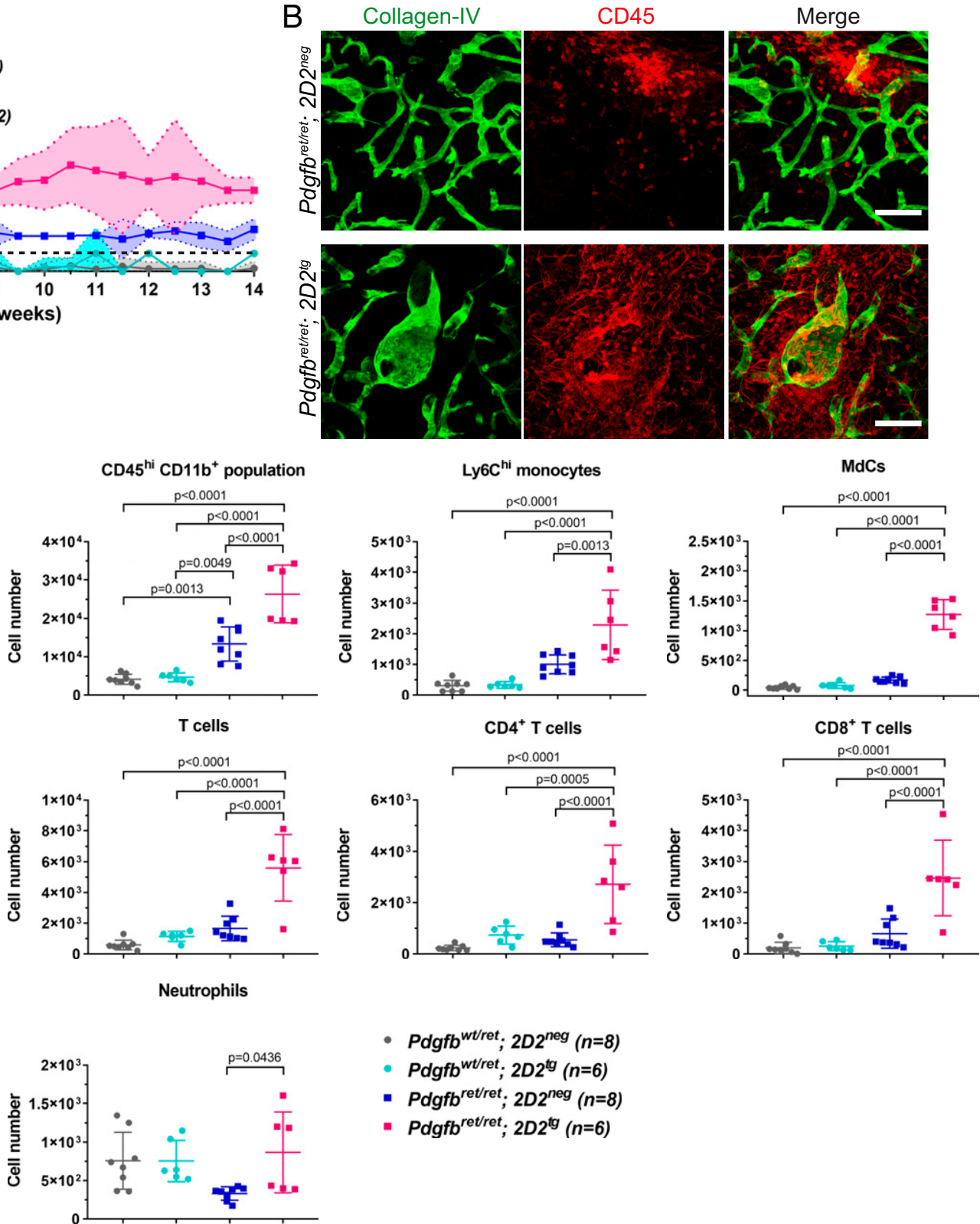

- $P d g f b^{\text {wt/ret }} ; 2 D 2^{\text {neg }}$ ( $n=8$ )

- Pdgfb ${ }^{\text {wt/ret }} ; 2 D 2^{\text {tg }}(n=6)$

- Pdgfb ${ }^{\text {ret/ret }} ; 2 D 2^{\text {neg }}(n=8)$

- Pdgfb ${ }^{\text {ret/ret }} ; 2 D 2^{\text {tg }}$ (n=6)

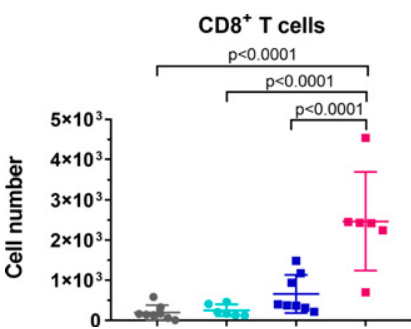

Fig. 7. Pericyte-deficient mice expressing myelin-specific TCR develop neurological symptoms accompanied by immune cell infiltration. (A) Scoring of cerebellar ataxia in Pdgfb ${ }^{\text {ret/ret }} \times \mathrm{Pdgfb}^{\text {wt/ret}} ; 2 \mathrm{D} 2^{\text {tg }}$ crossing offspring. Dashed black line indicates the mean baseline score of control mice (ataxia score 1 , occasional slip during the ledge test; $n=7-11$ mice per genotype). (B) Immunofluorescent detection of CD45 ${ }^{\text {hi }}$ leukocyte infiltrates and microglia (in red) in the striatum in Pdgfb ${ }^{\text {ret/ret }} ; 2 D 2^{\text {neg }}$ and Pdgfb ${ }^{\text {ret/ret }} ; 2 D 2^{\text {tg }}$ mice. Blood vessels are in green (collagen IV). (C) Representative flow cytometry pseudocolor plots showing the manual gating of microglia and other immune cell populations in the brain. $(C$ ) Quantification of absolute cell numbers of immune cells (gated as shown in SI Appendix, Fig. S13A) in the brains of control mice and Pdgfb ${ }^{\text {ret/ret }} ; 2 D 2^{\text {neg }}$ and $P d g f b^{\text {ret/ret }} ; 2 D 2^{\text {tg }}$ animals. Pdgfb ${ }^{\text {ret/ret }} ; 2 D 2^{\text {tg }}$ mice were euthanized at the peak of cerebellar ataxia symptoms (ataxia score of 6-9). Age of animals was 2-3 mo (C). Pooled data from four individual experiments. Data are presented as the mean \pm SD. One-way ANOVA followed by Tukey's post hoc test was used to determine the statistical significance. (Scale bars: $100 \mu$ m.)

natalizumab, a humanized monoclonal antibody against $\alpha 4$-integrin on leukocytes, has been proven to be an effective treatment in MS (25). However, no treatment blocking leukocyte entry on the endothelial side has been approved as of today in the therapy of MS. Treatment of $P d g f b^{\text {retret }}$ mice with a mix of anti-VCAM-1 and anti-ICAM-1 ameliorated EAE, rescued the mortality of pericytedeficient mice with $75 \%$ efficacy, and reduced the number of infiltrated leukocytes. These findings corroborate that the proinflammatory phenotype of the brain endothelium of pericyte-deficient mice substantially contributes to the massive leukocyte infiltration during neuroinflammation. However, combined treatment was not sufficient to abolish symptoms of pericyte-deficient mice, and it is likely that additional changes at the NVU induced by the absence of pericytes might contribute to the observed severe phenotype. Endothelial cells up-regulate Angiopoietin 2 (Angpt2) in the absence of pericytes $(1,2)$, and neutralizing antibodies to Angpt2 reduce vascular leakage and microaneurysm formation in the retina caused by pericyte deficiency (26). Blocking of Angpt2 also ameliorates EAE by suppressing vascular inflammation and leakage of plasma proteins (27). Thus, it is plausible that a deregulated Ang2-Tie2 signaling axis in endothelial cells in the absence of pericytes contributes to the aggravated neuroinflammation in $P d g f b^{\text {retret }}$ mice. Astrocyte end-feet 
serve yet another barrier limiting leukocyte entry during neuroinflammation $(28,29)$. Pericyte deficiency leads to a polarization defect of astrocyte end-feet (1), which may also facilitate the increased leukocyte trafficking in the absence of pericytes. Also, the vascular basement membrane limits the entry of autoreactive $T$ cells into the CNS (30), and, since pericytes secrete and deposit components of the vascular basement membrane (15), subtle alterations in the organization and composition of the vascular basement membrane could weaken its barrier function. In addition, increased vascular permeability to plasma proteins (e.g., fibrinogen) due to increased transcytosis in pericyte-deficient mice could contribute to leukocyte trafficking. Thus, the mechanism by which pericytes control leukocyte extravasation is likely multifaceted, and the increased leukocyte trafficking and aggravated neuroinflammation in pericyte-deficient mice is caused by a combination of changes in acellular (basement membrane, increased presence of plasma proteins in the brain parenchyma) and cellular components (endothelium, astrocytes) of the NVU (SI Appendix, Fig. S14A).

Similar persistent inflammation in the absence of pericytes as in the brain vasculature in $P d g f b^{\text {ret/ret }}$ mice has been described in the retina (26, 31-33). Published data on whether an acute dropout of pericytes in the adult organism leads to altered BBB permeability and alters the permissiveness of vasculature to leukocyte trafficking are conflicting and need further study $(26,34)$. It has been observed that CNS vasculature compensates to a certain extent for pericyte loss and reduced pericyte coverage. Upon pericyte loss in the brain, the area covered by a single pericyte can be filled by spatial rearrangement of nearby pericytes (35). Increased BBB permeability to intravenous tracers in juvenile $P d g f b^{F 7 / F 7}$ and $P d g f b^{F 7 /-}$ mice that have a $50-60 \%$ reduction in pericyte coverage is absent in adult animals (2), and up to $50 \%$ reduction in pericyte vessel coverage in adult mice does not lead to overt BBB permeability changes (1) (SI Appendix, Fig. S14B). The loss of pericytes may be compensated by other cells of the NVU such as astrocytes, which have been shown to promote BBB integrity and CNS immune quiescence $(28,36)$. Thus, in adult vasculature, which has established structures (e.g., basement membrane and astrocyte end-feet), pathological changes upon pericyte loss may develop gradually over time. Notably, an increased infiltration of leukocytes into the brain parenchyma has been reported in mouse pups that express one allele of constitutively active PDGFRB, which alters pericyte differentiation (37), indicating that both altered pericyte numbers/vessel coverage and activation state $(37,38)$ disturb pericyte-endothelial signaling. In the normal CNS, this disturbed signaling limits immune surveillance.

Increased infiltration of immune cells into the brain parenchyma in adult $P d g f b^{\text {ret/ret }}$ is not accompanied by demyelination. This finding indicates that the permissive state of the brain vasculature to immune cell entry is not sufficient to trigger demyelinating CNS pathology. Normal oligodendrocyte differentiation and myelination of adult $P d g f b^{\text {ret/ret }}$ mice been reported in a recent study (39). The findings of our study contradict a previous report of white matter changes and loss of myelin in another mouse model of pericyte deficiency, $P d g f r b^{F 7 / F 7}$ mice (40). Whereas pericytes and pericytelike cells have been shown to proliferate and promote oligodendrocyte differentiation during remyelination after acute CNS injury (39), mechanisms other than pericyte loss (i.e., dysfunction of neural cell types expressing PDGFRB) in old Pdgfrb mutants could contribute to the reported white matter changes during aging (40).

Cellular composition of infiltrated leukocytes could modify the clinical course of neuroinflammation in pericyte-deficient mice. Brain-restricted neuroinflammation is dominated by myeloid cells $\left(\mathrm{CD} 45^{\text {hi }} \mathrm{CD} 11 \mathrm{~b}^{+}\right.$, approximately $\left.87 \%\right)$ in $P d g f^{\text {ret }}$ ret mice during EAE. Increased number of myeloid cells could lead to an aggravated neuroinflammatory phenotype in $P d g f b^{\text {rettret }}$ mice. Increasing evidence points to the key role of myeloid cell subsets in mediating tissue damage in EAE and MS (reviewed in refs. 41, 42). Infiltration of CCR $2^{+}$Ly $6 \mathrm{C}^{\text {hi }}$ inflammatory monocytes from the blood into the CNS parenchyma coincides with the onset of the clinical signs and worsens the severity of EAE (43-46).

The localization of demyelinating lesions in MS patients shows a variable pattern. Typical affected areas are cerebral white matter (e.g., periventricular, corpus callosum), brainstem, and cerebellum (47). In the case of neuromyelitis optica spectrum disorders, another type of autoimmune demyelinating disease, the optic nerve and spinal cord are preferentially damaged (48). In EAE, a "classical" spinal cord phenotype (ascending flaccid paralysis) can be distinguished from "atypical" EAE with inflammation localized to the cerebrum, cerebellum, and brainstem. The underlying mechanisms leading to regional differences in leukocyte extravasation are not well understood. It has been suggested that the brain and spinal cord are distinct microenvironments with a distinct inflammatory cell repertoire, including different $\mathrm{T}$ cell types and different cytokines (49-52). We found that, upon induction of autoimmune neuroinflammation, $P d g f b^{\text {ret } / \text { ret }}$ mice succumb to an atypical EAE. In addition, $P d g f b^{\text {ret } r e t} ; 2 D 2^{t g}$ animals presented fluctuating symptoms of cerebellar ataxia, milder than after the induction of EAE. Histological and flow cytometry analyses of infiltrated immune cells, which showed neuroinflammation localized to the brain in pericyte-deficient mice, were in concordance with the clinical deficits. We showed that, although the vessel pericyte coverage is reduced in the spinal cord of $P d g f b^{\text {rettret }}$ mice compared with controls, pericyte vessel coverage in the spinal cord in $P d g f b^{\text {ret/ret }}$ mice is more complete compared with the brain $(1,17)$. This relatively spared vasculature of spinal cord vessels with a higher pericyte coverage and relatively small upregulation of ICAM-1 and VCAM$1(\sim 3-4 \%)$ compared with brain vessels could explain the preferential location of neuroinflammation in the brain in $P d g f b^{\text {retret }}$ and $P d g f b^{\text {ret/ret }} ; 2 D 2^{\text {tg }}$ mice.

$\mathrm{BBB}$ breakdown, one of the pathological hallmarks of MS (53, 54 ), is an early event in the formation of the inflammatory lesions and has been suggested to precede parenchymal inflammation (55). Interestingly, one of the early changes after induction of EAE, increased transcytosis of brain capillary endothelial cells, leads to increased vessel permeability (56). Pericyte damage (e.g., lipofuscin accumulation, membrane protrusions) has been described in chronic-progressive MS lesions (57). It has been suggested that the BBB becomes disrupted by early inflammatory microlesions via IL-1 $\beta$ (58) produced by MdCs and neutrophils after EAE induction (59). However, the nature of changes at the NVU that precede immune cell entry and cause failure of vascular immune regulatory function in MS is not known. Our study shows that intrinsic changes in the brain vasculature in mice facilitate the neuroinflammatory cascade and can influence the localization of the neuroinflammatory lesions. In the future, it would be interesting to investigate early changes in the brain vasculature of MS patients and determine whether vascular alterations regulate the localization of MS lesions. Since vascular dysfunction modulates leukocyte entry and neuroinflammation, vasoprotective therapies combined with preexisting treatments could lead to improved clinical outcomes in MS.

\section{Materials and Methods}

Transgenic mouse lines, reagents, immunohistochemistry and histochemistry, flow cytometry and data analysis, induction of EAE and scoring, FTY-720, anti-VCAM-1 and anti-ICAM-1 treatment, quantification of immunohistochemical and histochemical stainings, transmission electron microscopy, leukocyte isolation from tissues, and statistical analysis are described in SI Appendix, Materials and Methods.

Data Availability All relevant data are included in the article and/or SI Appendix.

ACKNOWLEDGMENTS. Samples for electron microscopic imaging were prepared by the Center for Microscopy and Image Analysis of the University of Zürich. Imaging was performed with equipment maintained by the Center for Microscopy and Image Analysis of the University of Zürich. Flow cytometry 
analysis was performed with equipment maintained by the Flow Cytometry Facility of the University of Zürich. We thank Dilay Cansever for help with flow cytometry analysis. This work was supported by grants from the Swiss National Science Foundation (Grants 31003A_159514 and 310030_188952 to A.K. and PP00P3_170626 and BSGI0_155832 to M.G.), the European Research Council

1. A. Armulik et al., Pericytes regulate the blood-brain barrier. Nature 468, 557-561 (2010).

2. R. Daneman, L. Zhou, A. A. Kebede, B. A. Barres, Pericytes are required for bloodbrain barrier integrity during embryogenesis. Nature 468, 562-566 (2010).

3. K. Stark et al., Capillary and arteriolar pericytes attract innate leukocytes exiting through venules and 'instruct' them with pattern-recognition and motility programs. Nat. Immunol. 14, 41-51 (2013).

4. D. Proebstl et al., Pericytes support neutrophil subendothelial cell crawling and breaching of venular walls in vivo. J. Exp. Med. 209, 1219-1234 (2012).

5. J. Hong et al., Role of tumor pericytes in the recruitment of myeloid-derived suppressor cells. J. Natl. Cancer Inst. 107, djv209 (2015).

6. R. Daneman, The blood-brain barrier in health and disease. Ann. Neurol. 72, 648-672 (2012).

7. A. Keller, Breaking and building the wall: The biology of the blood-brain barrier in health and disease. Swiss Med. Wkly. 143, w13892 (2013).

8. D. Davalos, K. Akassoglou, Fibrinogen as a key regulator of inflammation in disease. Semin. Immunopathol. 34, 43-62 (2012).

9. R. M. Ransohoff, D. A. Hafler, C. F. Lucchinetti, Multiple sclerosis-a quiet revolution. Nat. Rev. Neurol. 11, 134-142 (2015).

10. J. K. Ryu et al., Blood coagulation protein fibrinogen promotes autoimmunity and demyelination via chemokine release and antigen presentation. Nat. Commun. 6, 8164 (2015).

11. E. E. Kwon, J. W. Prineas, Blood-brain barrier abnormalities in longstanding multiple sclerosis lesions. An immunohistochemical study. J. Neuropathol. Exp. Neurol. 53, 625-636 (1994)

12. S. Hochmeister et al., Dysferlin is a new marker for leaky brain blood vessels in multiple sclerosis. J. Neuropathol. Exp. Neurol. 65, 855-865 (2006).

13. J. I. Alvarez et al., Focal disturbances in the blood-brain barrier are associated with formation of neuroinflammatory lesions. Neurobiol. Dis. 74, 14-24 (2015).

14. B. Engelhardt, R. M. Ransohoff, Capture, crawl, cross: The T cell code to breach the blood-brain barriers. Trends Immunol. 33, 579-589 (2012).

15. M. Vanlandewijck et al., A molecular atlas of cell types and zonation in the brain vasculature. Nature 554, 475-480 (2018).

16. R. Harb, C. Whiteus, C. Freitas, J. Grutzendler, In vivo imaging of cerebral microvascular plasticity from birth to death. J. Cereb. Blood Flow Metab. 33, 146-156 (2013).

17. M. Vanlandewijck et al., Functional characterization of germline mutations in PDGFB and PDGFRB in primary familial brain calcification. PLoS One 10, e0143407 (2015).

18. R. Villaseñor et al., Trafficking of endogenous immunoglobulins by endothelial cells at the blood-brain barrier. Sci. Rep. 6, 25658 (2016)

19. A. Keller et al., Prion pathogenesis is unaltered in a mouse strain with a permeable blood-brain barrier. PLoS Pathog. 14, e1007424 (2018).

20. R. L. Terry, I. Ifergan, S. D. Miller, Experimental autoimmune encephalomyelitis in mice. Methods Mol. Biol. 1304, 145-160 (2016).

21. S. J. Guyenet et al., A simple composite phenotype scoring system for evaluating mouse models of cerebellar ataxia. J. Vis. Exp. 39, 1787 (2010).

22. S. Mandala et al., Alteration of lymphocyte trafficking by sphingosine-1-phosphate receptor agonists. Science 296, 346-349 (2002).

23. N. D. Lewis et al., Circulating monocytes are reduced by sphingosine-1-phosphate receptor modulators independently of S1P3. J. Immunol. 190, 3533-3540 (2013).

24. E. Bettelli et al., Myelin oligodendrocyte glycoprotein-specific T cell receptor transgenic mice develop spontaneous autoimmune optic neuritis. J. Exp. Med. 197, 1073-1081 (2003).

25. C. H. Polman et al.; AFFIRM Investigators, A randomized, placebo-controlled trial of natalizumab for relapsing multiple sclerosis. N. Engl. J. Med. 354, 899-910 (2006).

26. D. Y. Park et al., Plastic roles of pericytes in the blood-retinal barrier. Nat. Commun. 8 15296 (2017)

27. Z. Li et al., Angiopoietin-2 blockade ameliorates autoimmune neuroinflammation by inhibiting leukocyte recruitment into the CNS. J. Clin. Invest. 130, 1977-1990 (2020).

28. J. I. Alvarez et al., The Hedgehog pathway promotes blood-brain barrier integrity and CNS immune quiescence. Science 334, 1727-1731 (2011).

29. S. Horng et al., Astrocytic tight junctions control inflammatory CNS lesion pathogenesis. J. Clin. Invest. 127, 3136-3151 (2017).

30. X. Zhang et al., The endothelial basement membrane acts as a checkpoint for entry of pathogenic T cells into the brain. J. Exp. Med. 217, e20191339 (2020).

31. P. Lindblom et al., Endothelial PDGF-B retention is required for proper investment of pericytes in the microvessel wall. Genes Dev. 17, 1835-1840 (2003). under the European Union's Horizon 2020 Research and Innovation Programme (Grant 819229 to M.G.), the Synapsis Foundation and the Choupette Foundation (Grant 2019-PI02), the Swiss Heart Foundation, the Swiss Cancer League (Grant KLS-3848-02-2016 to A.K.), the Swiss Society of Multiple Sclerosis (to A.K. and B.S.), and the Leducq Foundation (Grant 14CVD02 to A.K. and M.H.H.)

32. M. Enge et al., Endothelium-specific platelet-derived growth factor-B ablation mimic diabetic retinopathy. EMBO J. 21, 4307-4316 (2002).

33. S. Ogura et al., Sustained inflammation after pericyte depletion induces irreversible blood-retina barrier breakdown. JCI Insight 2, e90905 (2017).

34. A. M. Nikolakopoulou et al., Pericyte loss leads to circulatory failure and pleiotrophin depletion causing neuron loss. Nat. Neurosci. 22, 1089-1098 (2019).

35. A. A. Berthiaume et al., Dynamic remodeling of pericytes in vivo maintains capillary coverage in the adult mouse brain. Cell Rep. 22, 8-16 (2018).

36. M. Segarra et al., Endothelial Dab1 signaling orchestrates neuro-glia-vessel communication in the central nervous system. Science 361, eaao2861 (2018).

37. L. E. Olson, P. Soriano, PDGFR $\beta$ signaling regulates mural cell plasticity and inhibits fat development. Dev. Cell 20, 815-826 (2011).

38. X. Duan et al., Cadherin combinations recruit dendrites of distinct retinal neurons to a shared interneuronal scaffold. Neuron 99, 1145-1154.e6 (2018).

39. A. G. De La Fuente et al., Pericytes stimulate oligodendrocyte progenitor cell differentiation during CNS remyelination. Cell Rep. 20, 1755-1764 (2017)

40. A. Montagne et al., Pericyte degeneration causes white matter dysfunction in the mouse central nervous system. Nat. Med. 24, 326-337 (2018).

41. M. K. Mishra, V. W. Yong, Myeloid cells-Targets of medication in multiple sclerosis. Nat. Rev. Neurol. 12, 539-551 (2016)

42. A. L. Croxford, S. Spath, B. Becher, GM-CSF in neuroinflammation: Licensing myeloid cells for tissue damage. Trends Immunol. 36, 651-662 (2015).

43. I. L. King, T. L. Dickendesher, B. M. Segal, Circulating Ly-6C+ myeloid precursors migrate to the CNS and play a pathogenic role during autoimmune demyelinating disease. Blood 113, 3190-3197 (2009).

44. M. K. Mishra, J. Wang, C. Silva, M. Mack, V. W. Yong, Kinetics of proinflammatory monocytes in a model of multiple sclerosis and its perturbation by laquinimod. $A m$. J. Pathol. 181, 642-651 (2012).

45. A. Mildner et al., CCR2+Ly-6Chi monocytes are crucial for the effector phase of autoimmunity in the central nervous system. Brain 132, 2487-2500 (2009).

46. B. Ajami, J. L. Bennett, C. Krieger, K. M. McNagny, F. M. Rossi, Infiltrating monocytes trigger EAE progression, but do not contribute to the resident microglia pool. Nat. Neurosci. 14, 1142-1149 (2011).

47. C. A. Dendrou, L. Fugger, M. A. Friese, Immunopathology of multiple sclerosis. Nat. Rev. Immunol. 15, 545-558 (2015).

48. S. Huda et al., Neuromyelitis optica spectrum disorders. Clin. Med. (Lond.) 19, 169-176 (2019).

49. I. M. Stromnes, L. M. Cerretti, D. Liggitt, R. A. Harris, J. M. Goverman, Differential regulation of central nervous system autoimmunity by $T(H) 1$ and $T(H) 17$ cells. Nat. Med. 14, 337-342 (2008)

50. J. S. Tzartos et al., Interleukin-17 production in central nervous system-infiltrating $\mathrm{T}$ cells and glial cells is associated with active disease in multiple sclerosis. Am. J. Pathol. 172, 146-155 (2008).

51. H. Kebir et al., Human TH17 lymphocytes promote blood-brain barrier disruption and central nervous system inflammation. Nat. Med. 13, 1173-1175 (2007).

52. H. S. Domingues, M. Mues, H. Lassmann, H. Wekerle, G. Krishnamoorthy, Functional and pathogenic differences of Th1 and Th17 cells in experimental autoimmune en cephalomyelitis. PLoS One 5, e15531 (2010).

53. L. A. Stone et al., Blood-brain barrier disruption on contrast-enhanced MRI in patients with mild relapsing-remitting multiple sclerosis: Relationship to course, gender, and age. Neurology 45, 1122-1126 (1995)

54. G. G. Ortiz et al., Role of the blood-brain barrier in multiple sclerosis. Arch. Med. Res. 45, 687-697 (2014)

55. A. G. Kermode et al., Breakdown of the blood-brain barrier precedes symptoms and other MRI signs of new lesions in multiple sclerosis. Pathogenetic and clinical implications. Brain 113, 1477-1489 (1990).

56. L. Claudio, Y. Kress, W. T. Norton, C. F. Brosnan, Increased vesicular transport and decreased mitochondrial content in blood-brain barrier endothelial cells during experimental autoimmune encephalomyelitis. Am. J. Pathol. 135, 1157-1168 (1989).

57. L. Claudio, C. S. Raine, C. F. Brosnan, Evidence of persistent blood-brain barrier abnormalities in chronic-progressive multiple sclerosis. Acta Neuropathol. 90, 228-238 (1995).

58. A. T. Argaw et al., IL-1beta regulates blood-brain barrier permeability via reactivation of the hypoxia-angiogenesis program. J. Immunol. 177, 5574-5584 (2006).

59. S. A. Lévesque et al., Myeloid cell transmigration across the CNS vasculature triggers IL-1 $\beta$-driven neuroinflammation during autoimmune encephalomyelitis in mice. J. Exp. Med. 213, 929-949 (2016). 